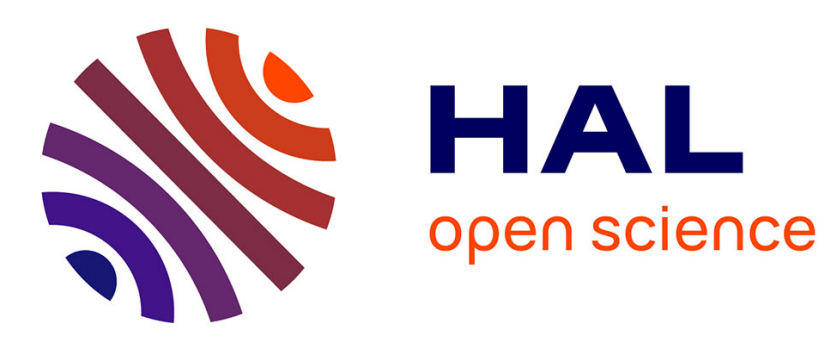

\title{
A mathematical model of leptin resistance
}

Marine Jacquier, Hédi A. Soula, Fabien Crauste

\section{To cite this version:}

Marine Jacquier, Hédi A. Soula, Fabien Crauste. A mathematical model of leptin resistance. Mathematical Biosciences, 2015, 267, 10.1016/j.mbs.2015.06.008 . hal-01233483

\section{HAL Id: hal-01233483 \\ https://inria.hal.science/hal-01233483}

Submitted on 25 Nov 2015

HAL is a multi-disciplinary open access archive for the deposit and dissemination of scientific research documents, whether they are published or not. The documents may come from teaching and research institutions in France or abroad, or from public or private research centers.
L'archive ouverte pluridisciplinaire $\mathbf{H A L}$, est destinée au dépôt et à la diffusion de documents scientifiques de niveau recherche, publiés ou non, émanant des établissements d'enseignement et de recherche français ou étrangers, des laboratoires publics ou privés. 


\title{
A mathematical model of leptin resistance*
}

\author{
Marine Jacquier ${ }^{1,2}$, Hedi A. Soula ${ }^{3,4}$, Fabien Crauste ${ }^{1,2}$
}

${ }^{1}$ Université de Lyon, Université Claude Bernard Lyon 1, CNRS UMR 5208, Institut Camille Jordan, 43 blvd. du 11 novembre 1918, F-69622 Villeurbanne cedex, France

${ }^{2}$ Inria Team Dracula, Inria Center Grenoble Rhône-Alpes, France

${ }^{3}$ CarMeN, INSERM U1060, Université de Lyon, INSA-Lyon, Univ. Lyon-1, F-69621, Villeurbanne, France

${ }^{4}$ Inria Team Beagle, Inria Center Grenoble Rhône-Alpes, France

\begin{abstract}
Obesity is often associated with leptin resistance, which leads to a physiological system with high leptin concentration but unable to respond to leptin signals and to regulate food intake. We propose a mathematical model of the leptin-leptin receptors system, based on the assumption that leptin is a regulator of its own receptor activity, and investigate its qualitative behavior. Based on current knowledge and previous models developed for body weight dynamics in rodents, the model includes the dynamics of leptin, leptin receptors and the regulation of food intake and body weight. It displays two stable equilibria, one representing a healthy state and the other one an obese and leptin resistant state. We show that a constant leptin injection can lead to leptin resistance and that a temporal variation in some parameter values influencing food intake can induce a change of equilibrium and a pathway to leptin resistance and obesity.
\end{abstract}

\section{Introduction}

Obesity is characterized by an excessive accumulation of adipose tissue resulting from an energy imbalance, where energy intake exceeds energy expenditure. Obesity has an important impact on health, with an increased mortality and is often associated with an increased risk of diseases including diabetes and hypertension [13]. Causes of this dysregulation, including genetic and environmental conditions, and possible treatments are widely investigated [20,33].

Obesity is associated in most human and rodent cases to high concentrations of leptin in plasma $[13,32,39,50]$. Leptin is a hormone produced by adipocytes (fat cells), regulating food intake and energy expenditure, known for its role as an indicator of the amount of fat storage in the organism $[12,15]$ : leptin concentration is proportional to fat mass. Increased leptin induces an inhibition of food intake. Leptin is thus involved in mechanisms regulating body weight [14].

Food intake is a complex process, regulated by a wide variety of oral and post-oral signals [29, 30, 40], including hormones ghrelin [47], cholecystokinin [10] and leptin [14] to name a few (see for instance [8] for details on food intake regulation by hormones). Leptin is known as the main regulator of food intake and energy expenditure. Circulating in plasma, leptin crosses the blood-brain barrier and reaches the arcuate nucleus of the hypothalamus where it binds to specific receptors. Activation of leptin receptors by leptin induces signaling cascades which negatively regulate food intake. Leptin also seems to influence its own impact on food intake regulation by regulating the expression of its cognate receptors [28, 31, 35, 46, 49] (see Figure 1 for a schematic representation).

There exist different types of leptin receptors [43], in particular LepRa and LepRb [4]. LepRa receptors may play a role in the transport of leptin from plasma to cerebrospinal fluid $[3,17,27]$.

\footnotetext{
${ }^{*}$ This work has been published in Mathematical Biosciences: Marine Jacquier, Hédi A. Soula, Fabien Crauste, A mathematical model of leptin resistance, Mathematical Biosciences, Volume 267, September 2015, Pages 10-23, ISSN 0025-5564, http://dx.doi.org/10.1016/j.mbs.2015.06.008. (http://www.sciencedirect.com/science/article/pii/S0025556415001261)
} 
LepRb receptors are located in the hypothalamus and are responsible for activation of the food intake and energy expenditure regulation pathways [1, 2].

Leptin resistance corresponds to the system's inability to integrate leptin signals in food intake regulation. The resistance can occur either at the blood-brain barrier (leptin transport to the brain is reduced [5]) or in the hypothalamus (reduced amount of receptors in the hypothalamus [11]). Leptin resistance has been observed in obese individuals: whereas intravenous injections of leptin lead to a decrease in food intake in healthy subjects, less important decrease or no decrease at all are observed in obese individuals [45]. When the resistance is located in the hypothalamus, an injection of leptin directly into the cerebrospinal fluid is not able to reduce food intake [21]. Leptin resistance is then characterized by high concentrations of leptin in the brain that lead to a decrease in leptin receptors in the hypothalamus, the system has then a lower sensitivity to leptin and does not regulate food intake as well as it should. It results in increased food consumption and increased body fat, which produces more and more leptin, leading to a vicious cycle and in some cases, to the development of obesity [38, 50].

The purpose of this work is to propose a theoretical model of leptin resistance development, and to qualitatively study the dynamics behind the development of leptin resistance and its influence on food intake and body weight. To our knowledge, there is no mathematical model describing the emergence of leptin resistance. However, there exist a wide variety of models describing the regulations of body weight and metabolism (see [9] and the references therein, [7, 24]). These models, based mainly on ordinary differential equations, consider different mechanisms of regulation such as energy use in humans $[6,23,24]$ or in rodents $[18,19]$. In humans, Horgan [24] described the regulation of body weight regulated only by itself and food intake with a discrete stochastic model and concluded that body weight remains around a fixed value if the mean food intake and physical activity are constant. In [7], Chow and Hall studied the impact of stochastic fluctuations in food intake on body weight evolution and concluded that short-term fluctuations in food intake have a limited impact on body-weight. Guo and Hall [18, 19] developed an ordinary differential equation model based on laws of energy conservation to predict changes in body weight and energy expenditure, using only energy intake, and applied this model to mice.

Other models considered the effects of hormones involved in food intake regulation in rodents [25, 42]. Tam et al. [42] proposed a model, based on a system of ordinary differential equations, of metabolic regulation by leptin and compared normal regulation to leptin resistance. The latter has been modeled as a modification in parameter values involved in the transport of leptin into the brain and in the regulation of food intake, yet the authors did not consider the emergence of leptin resistance. There also exist models describing insulin resistant systems, linked to the development of diabetes [16, 44]. Topp et al. [44] modeled the dynamics of blood glucose, insulin and $\beta$-cell mass, using a system of ordinary differential equations, and studied perturbations, such as insulin resistance, that can lead to diabetes.

Our objective is to present a simple theoretical model of leptin resistance, taking into account leptin concentration, leptin receptors density, food intake and body weight. Based on previous models of body weight dynamics in rodents $[18,19]$, the main assumption of our model will be that leptin both up and down regulates leptin receptor expression - by positively regulating receptor degradation, and by also positively regulating receptor production. Activation of leptin receptors by leptin leads to the regulation of food intake, which influences body weight evolution. Leptin resistance is characterized by a state of the system with a high level of leptin which does not induce a loss of fat mass. The development of leptin resistance corresponds to the dynamical evolution of the system from a healthy state to a leptin resistant state. We study the existence of equilibria of the system and analyze their stability. We describe the model qualitative behavior in steady conditions and for different cases of biologically relevant perturbations, such as an injection of leptin or modifications of parameter values, which can lead to the development of leptin resistance and obesity. 

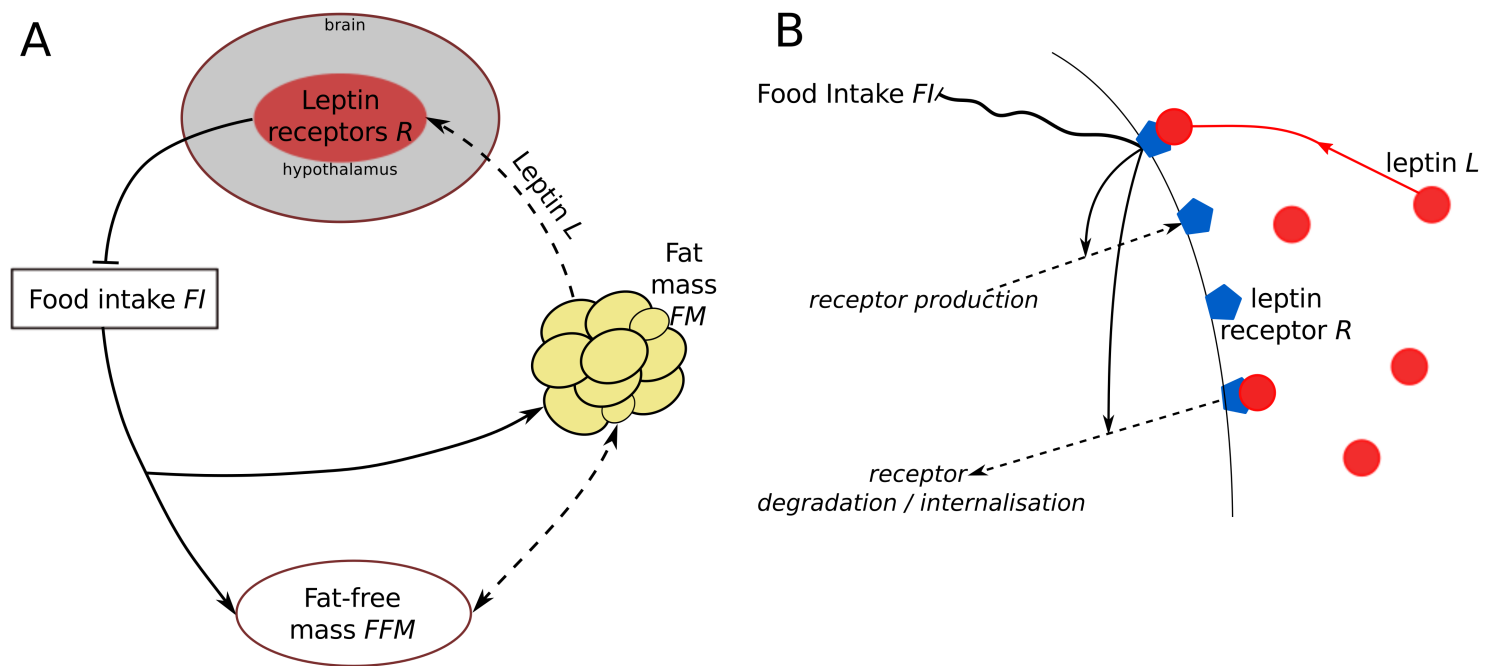

Figure 1: A. Schematic representation of the leptin-mediated regulation of food intake at the scale of the whole system. Lines represent the action of the source on the target: straight lines with arrows display positive actions, bar-headed lines display inhibitions and dashed lines display actions that can be positive or negative depending on the parameter and variable values. B. Action of leptin (in red) on receptors (in blue) in the hypothalamus. Leptin binding on its receptors inhibits food intake (see A) and triggers a regulation of the production and degradation of receptors [28, 31, 35, 46, 49].

\section{Methods}

\subsection{Mathematical model}

In this section, the mathematical model is described. It focuses on fat mass, fat-free mass and food intake evolution and takes into account plasma leptin and leptin receptor dynamics to mediate food intake. Table 1 provides a list of variables and their units, Table 2 a a list of parameters, and Figure 1 a schematic representation of the system.

We present below the equations of our model and the assumptions that led to this model. The main assumptions are listed hereafter:

(A1) Fat mass and fat-free mass dynamics follow the experiment-based relationship in mice described in Guo and Hall [18, 19].

(A2) Energy expenditure is supposed to be a linear function of fat and fat-free mass [25, 34].

(A3) Leptin is produced proportionally to fat mass [42].

(A4) Leptin concentration regulates leptin receptors dynamics (it impacts both their production and their degradation) $[28,49]$.

(A5) Leptin-mediated activation of leptin receptors is described in terms of occupation theory (Hill function with a maximal response proportional to the density of receptors) [26, 36, 41].

(A6) Activated leptin receptors are the main regulators of food intake [31].

Fat mass $F M$ (in grams) and fat-free mass $F F M$ (in grams) dynamics are adapted from the standard model of fat-free and fat mass dynamics proposed by Guo and Hall [18, 19]. This model describes changes in body composition as a function of energy dynamics in mice, based on fitting of experimental data (Assumption (A1)). Variations of $F M$ and $F F M$ are correlated with the difference between energy intake $(E I)$ and energy expenditure $(E E), E I-E E\left(k c a l . \mathrm{min}^{-1}\right)$, which is a function of fat mass, fat-free mass and food intake (see Equation (3)) [25], as follows

$$
\frac{\mathrm{d} F M}{\mathrm{~d} t}=\frac{E I-E E}{\rho_{F F M} \Omega+\rho_{F M}},
$$




\begin{tabular}{|lc|c|}
\hline Variable & $F M$ & Unit \\
\hline \hline Fat mass & $F F M$ & $g$ \\
\hline Fat-free mass & $L$ & $n g . m L^{-1}$ \\
\hline Plasma leptin concentration & $R$ & $m o l . L^{-1}$ \\
\hline Density of leptin receptors & $F I$ & $g$ \\
\hline Food intake & r \\
\hline
\end{tabular}

Table 1: Variables of the model, their notations and units.

\begin{tabular}{|c|c|c|}
\hline Parameter & Unit & Default value \\
\hline$\gamma_{L}$ & $\bar{c}^{n g} \cdot m L^{-1} \cdot g^{-1} \cdot \mathrm{min}^{-1}$ & 0.5 \\
\hline$\delta_{L}$ & min $^{-1}$ & 0.8 \\
\hline$\gamma_{R}$ & mol. $L^{-1} \cdot$ min $^{-1}$ & 2.5 \\
\hline$\delta_{R}$ & min $^{-1}$ & 0.9 \\
\hline$\lambda_{R 1}$ & $m L \cdot n g^{-1}$ & 0.21 \\
\hline$\lambda_{R 2}$ & $m L^{2} \cdot n g^{-2}$ & 0.05 \\
\hline$\delta_{F I}$ & min $^{-1}$ & 1.2 \\
\hline$\gamma_{F I}$ & g.min $^{-1}$ & 2.3 \\
\hline$\phi$ & L. $\mathrm{mol}^{-1}$ & 1 \\
\hline$\theta$ & $n g \cdot m L^{-1}$ & 3 \\
\hline$n$ & N.U. & 2 \\
\hline$\gamma_{\Omega}$ & N.U. & 0.003 \\
\hline$\alpha$ & N.U. & 0.005 \\
\hline$\kappa$ & $g^{-1}$ & 0.05 \\
\hline$\gamma_{E}$ & kcal.g $g^{-1}$. min $^{-1}$ & 0.14 \\
\hline$\eta$ & $\min ^{-1}$ & 0.00012 \\
\hline$\rho_{F F M}$ & $k c a l . g^{-1}$ & 1.8 \\
\hline$\rho_{F M}$ & $k c a l . g^{-1}$ & 9.4 \\
\hline$\xi$ & $k c a l$ & 400 \\
\hline
\end{tabular}

Table 2: Parameter units and default values used in the simulations. Parameter values have been chosen in order to characterize bistability. N.U. denotes "non-dimensional unit".

$$
\frac{\mathrm{d} F F M}{\mathrm{~d} t}=\frac{\Omega(E I-E E)}{\rho_{F F M} \Omega+\rho_{F M}},
$$

where parameters $\rho_{F F M}$ and $\rho_{F M}$ denote the caloric densities of fat-free mass and fat mass respectively. Energy intake corresponds to the caloric content of food intake FI characterized by the caloric density $\gamma_{E}$ of the food. Following (A2), energy expenditure is assumed to be proportional to fat mass and fat-free mass with a basal energy expenditure $\xi$ and a rate of energy expenditure $\eta[25,34]$. The energy balance is then defined as

$$
E I-E E=\gamma_{E} F I-\eta\left(\rho_{F F M} F F M+\rho_{F M} F M+\xi\right) .
$$

The function $\Omega$ in (1)-(2) denotes the body composition function, describing the relationship between fat mass and fat-free mass. The expression of $\Omega$ has been deduced from experimental data $[18,19]$, and is given by

$$
\Omega:=\frac{\mathrm{d} F F M}{\mathrm{~d} F M}=\gamma_{\Omega}(1+\alpha \exp (\kappa F M)),
$$

characterized by parameters $\gamma_{\Omega}, \alpha$ and $\kappa$.

Fat-free mass FFM can be explicitly obtained from fat mass $F M$ and initial conditions, by using (4). One obtains

$$
F F M=\frac{\gamma_{\Omega}(\kappa F M+\alpha \exp (\kappa F M))}{\kappa}+C,
$$


with

$$
C:=F F M(0)-\gamma_{\Omega}\left(F M(0)+\frac{\alpha}{\kappa} \exp (\kappa F M(0)) .\right.
$$

Hence, from (3), EI - EE can be expressed as a function of $F I$ and $F M$ only, as follows

$$
E I-E E=\gamma_{E} F I-\eta\left(\left(\rho_{F M}+\rho_{F F M} \gamma_{\Omega}\right) F M+\frac{\rho_{F F M} \gamma_{\Omega} \alpha}{\kappa} \exp (\kappa F M)+\rho_{F F M} C+\xi\right) .
$$

Plasma leptin $L$ (in $n g . m L^{-1}$ ) is produced by adipocytes proportionally to fat mass [25, 42] (Assumption (A3)), so

$$
\frac{\mathrm{d} L}{\mathrm{~d} t}=\gamma_{L} F M-\delta_{L} L,
$$

where $\gamma_{L}$ is the rate of leptin production and $\delta_{L}$ the rate of leptin degradation (via renal elimination $[25,42])$.

Let denote by $R$ (mol. $L^{-1}$ ) the density of leptin receptors located in the hypothalamus, which mediate the inhibition of food intake by leptin. Leptin receptors expression is regulated by leptin $[28,31,35,38,45,46,49]$ (Assumption (A4)). We assume that both production and degradation of $R$ are increased by leptin $L$, and we account for basal production $\left(\gamma_{R}\right)$ and degradation $\left(\delta_{R}\right)$ rates. Thus, the number of receptors will at first increase with leptin, then decrease when the concentration of leptin is high. It must be noted however that positive regulation of leptin receptor production by leptin is not necessary to obtain the results presented in Section 3. The density of receptors then evolves according to the following equation,

$$
\frac{\mathrm{d} R}{\mathrm{~d} t}=\gamma_{R}\left(1+\lambda_{R 1} L\right)-\delta_{R}\left(1+\lambda_{R 2} L^{2}\right) R .
$$

Parameters $\lambda_{R 1}$ and $\lambda_{R 2}$ characterize the effect of leptin on the production $\left(\lambda_{R 1}\right)$ and degradation $\left(\lambda_{R 2}\right)$ of leptin receptors. Our main assumption is that the influence of leptin on degradation is more important than on production for high leptin concentrations. This assumption is satisfied, for instance, in the absence of positive regulation of receptor production (when $\lambda_{R 1}=0$ ), as soon as the degradation rate of receptors is an increasing function of leptin concentration, which is in agreement with the literature $[28,49]$. One may note that other leptin-dependent functions could be used for the description of production and degradation of receptors, provided that they satisfy the previously mentioned assumption. Our choice has been motivated by the will to obtain a simple, in terms of parameter number and dynamics, yet general model. It has been inspired by Topp et al. [44], who used a similar function to model the dynamics of $\beta$-cell mass.

Activation of leptin receptors in the hypothalamus leads to a pathway controlling food intake $F I$ (in grams) [31]. The response $\Phi_{R}(L)$ of the activation of leptin receptors can be described in terms of occupation theory $[26,36,41]$ by a Hill function (Assumption (A5)), where the maximal response is proportional to the density of receptors, given by

$$
\Phi_{R}(L)=\frac{\phi R L^{n}}{L^{n}+\theta^{n}},
$$

with $\phi R$ the maximal response, $\theta$ a threshold corresponding to $50 \%$ of activation and the integer $n \geq 1$ a sensitivity coefficient.

Finally according to Assumption (A6), we assume food intake $F I$ is inhibited by the activation of leptin receptors $[14,31]$, so

$$
\frac{\mathrm{d} F I}{\mathrm{~d} t}=\frac{\gamma_{F I}}{1+\Phi_{R}(L)}-\delta_{F I} F I,
$$

where $\gamma_{F I}$ describes the rate of stimulation of food intake, and $\delta_{F I}$ denotes an inhibition rate of food intake.

The system formed with equations (1), (6), (7), (8) and (9) describes the interactions between food intake and fat mass mediated by leptin and leptin receptors dynamics (see Figure 2 for a state variable flow diagram). In the following, we focus on the dynamics of this system of equations, given by: 


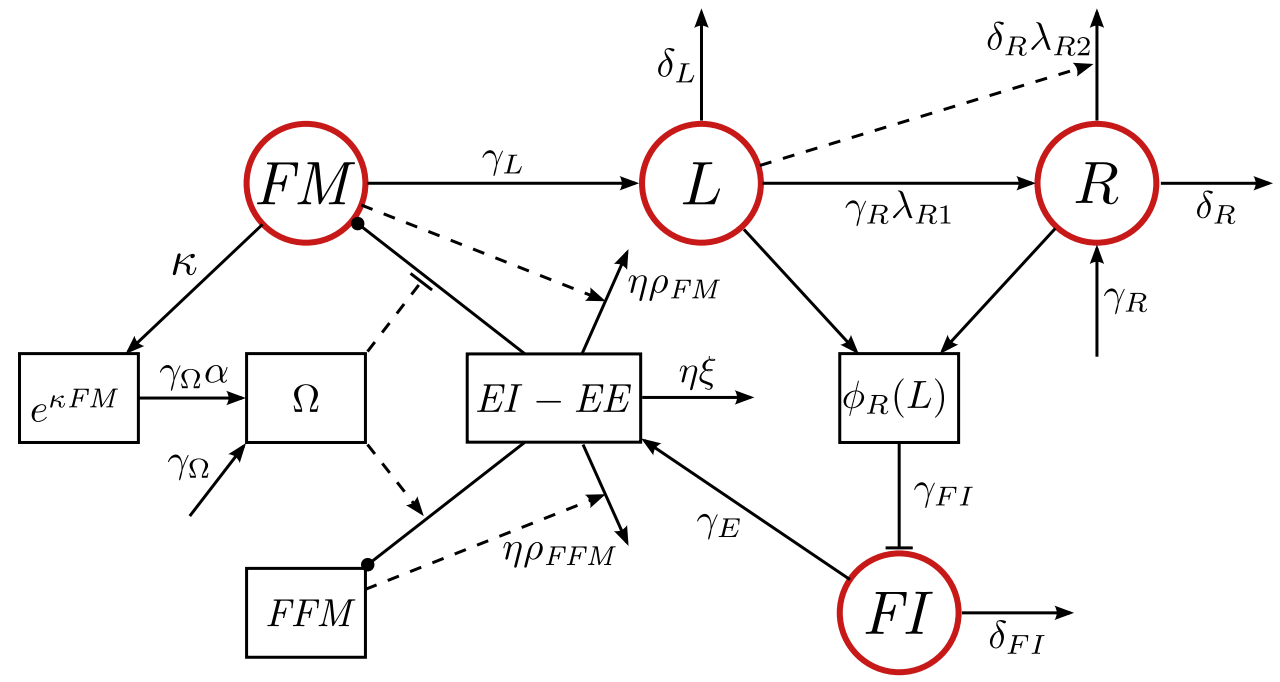

Figure 2: State variable flow diagram of System (10). Main variables are displayed in red circles. Arrows indicate a positive contribution, bar-headed lines a negative contribution and dot-ended lines indicate a contribution that can be either positive or negative depending on the value of the considered quantity (here, only $E I-E E$, the energy balance, can positively contribute to fat mass and fat-free mass in some conditions, and negatively in other conditions). Straight lines represent either production or degradation of variables, while dashed lines represent the influence of one variable on a biological process.

$$
\left\{\begin{aligned}
\frac{\mathrm{d} F M}{\mathrm{~d} t}= & \frac{\gamma_{E} F I}{\rho_{F F M} \gamma_{\Omega}(1+\alpha \exp (\kappa F M))+\rho_{F M}} \\
& -\frac{\eta\left(\left(\rho_{F M}+\rho_{F F M} \gamma_{\Omega}\right) F M+\frac{\rho_{F F M} \gamma_{\Omega} \alpha}{\kappa} \exp (\kappa F M)+\rho_{F F M} C+\xi\right)}{\rho_{F F M} \gamma_{\Omega}(1+\alpha \exp (\kappa F M))+\rho_{F M}} \\
\frac{\mathrm{d} L}{\mathrm{~d} t}= & \gamma_{L} F M-\delta_{L} L \\
\frac{\mathrm{d} R}{\mathrm{~d} t}= & \gamma_{R}\left(1+\lambda_{R 1} L\right)-\delta_{R}\left(1+\lambda_{R 2} L^{2}\right) R \\
\frac{\mathrm{d} F I}{\mathrm{~d} t}= & \frac{\gamma_{F I}\left(L^{n}+\theta^{n}\right)}{L^{n}(1+\phi R)+\theta^{n}}-\delta_{F I} F I .
\end{aligned}\right.
$$

Before presenting in the next section how we use this model to investigate the development of leptin resistance, let us briefly comment on the non-negativity of the solutions of System (10).

Variables $L, R$ and FI remain non-negative as long as the other variables involved in System (10) are positive, as expected. Solutions of the fat mass equation however can become negative under specific conditions. Basically, if $F M=0$ then $d F M / d t>0$ if and only if

$$
\gamma_{E} F I>\eta\left(\rho_{F F M}\left(\frac{\gamma_{\Omega} \alpha}{\kappa}+C\right)+\xi\right) .
$$

Consequently, when food intake FI is close to zero and fat mass is also close to zero then the difference between energy intake and energy expenditure can be negative and solutions can become negative. This is a property of the Guo and Hall's model [18, 19], which has been proposed to model fat and fat-free mass dynamics in either normal or obese states, associated with normal or high food intakes, but cannot account for an extreme situation corresponding to low food intake associated with low fat mass.

In order to ensure non-negativity of the solutions of System (10), we considered throughout this manuscript parameter values and initial conditions such that $E I-E E$ is positive for low fat mass, satisfying the assumptions in Guo and Hall $[18,19]$ and hence do not induce a loss of positivity for $F M$. 
System (10) will be used in Section 3 to investigate the development of leptin resistance and how it can be related to obesity, based on the variation of food intake stimulation as detailed hereafter.

\subsection{Varying food consumption}

In the previous sections, we considered all parameter values to be constant; however, the characteristics of a biological system evolve with time for a single individual. Parameters describing rates of creation and degradation (for example parameters $\delta_{L}$ and $\gamma_{L}$ in Equation (6)) or sensitivity, such as $\lambda_{R 1}$, are impacted by aging. Environmental conditions can also induce variations, for example environment can impact food consumption. We choose here to focus only on the variations in parameter $\gamma_{F I}$, representing the stimulation rate of food intake, and we investigate how variations in $\gamma_{F I}$ can induce first the development of leptin resistance and then obesity. Variability in parameter value also exists between individuals, explaining why some individuals are more susceptible than others to develop obesity even if they are submitted to similar changes.

To model progressive changes in food intake, we temporally modify the parameter $\gamma_{F I}$ in the following way

$$
\gamma_{F I}=\gamma_{F I}(t)=\gamma_{F I}^{0}+g(t),
$$

where $\gamma_{F I}^{0}$ represents the initial value of $\gamma_{F I}$ and $g(t)$ a temporal perturbation leading to an increase or a decrease in $\gamma_{F I}$. We assume that the modifications of food intake are negligible at short time scale (minutes, hours) and only impact the dynamics of the system after a long time (weeks, months). Indeed, modifications in food intake must be sustained for at least a few days to induce important metabolic modifications [25].

System (10) is either monostable or bistable depending on parameter values (see Section 3.1). When the system is bistable, we can define a healthy state corresponding to the equilibrium with low fat mass and an obese state corresponding to high fat mass. Without perturbations, the solutions of System (10) remain close to the healthy equilibrium. As the system's equilibria depend on parameter values, changes of equilibria values and bifurcations may occur when varying $\gamma_{F I}$ (see Section 3.1). As we are interested in pathways to leptin resistance and obesity, we assume in the following that initially System (10) is close to the healthy equilibrium, so initial conditions of $F M, L, R$ and FI are close to the healthy steady state values. Without perturbation, the system remains in this state.

We consider different patterns of food intake, characterized by different functions $g(t)$ (see Figure 3): a combination of two sigmoids (one increasing and the second one decreasing) and a sinusoid. These variations of $\gamma_{F I}$ induce a bypass of the regulation of food intake by leptin. We assume that this variations represent behavioral phenomena to regulate food intake and body weight.

The combination of two sigmoid functions corresponds to an increase in $\gamma_{F I}$ and then a decrease to a value lower (or higher) than the initial value. It is described by $g(t)$ with

$$
g(t)= \begin{cases}0, & \text { if } 0<t<t_{1}, \\ \Delta_{\gamma_{F I}^{1}} \frac{2 t^{3}-3\left(t_{1}+t_{2}\right) t^{2}+6 t_{1} t_{2} t+t_{1}^{2}\left(t_{1}-3 t_{2}\right)}{\left(t_{1}-t_{2}\right)^{3}}, & \text { if } t_{1}<t<t_{2}, \\ \Delta_{\gamma_{F I}^{1}}, & \text { if } t_{2}<t<t_{3}, \\ \Delta_{\gamma_{F I}^{1}}+\Delta_{\gamma_{F I}^{2}} \frac{-2 t^{3}+3\left(t_{3}+t_{4}\right) t^{2}-6 t_{3} t_{4} t-t_{3}^{3}+3 t_{3}^{2} t_{4}}{\left(t_{3}-t_{4}\right)^{3}}, & \text { if } t_{3}<t<t_{4}, \\ \Delta_{\gamma_{F I}^{1}}-\Delta_{\gamma_{F I}^{2}}, & \text { if } t>t_{4},\end{cases}
$$

where $\Delta_{\gamma_{F I}^{1}}$ is the amplitude of variation of the increasing sigmoid and $\Delta_{\gamma_{F I}^{2}}$ the amplitude of variation of the decreasing sigmoid. Parameters $t_{1}$ and $t_{2}$ define the increasing part of the function while $t_{3}$ and $t_{4}$ delimit the decreasing part. The plateau phase, associated with a high food intake stimulation equal to $\gamma_{F I}^{1}$, has a duration given by $t_{3}-t_{2}$. The final value $\gamma_{F I}^{2}$ resulting from the two variations can be higher or lower than the initial value $\gamma_{F I}^{0}$. This $\gamma_{F I}$ function represents a progressive increase in food consumption, which will stabilize at some point. The decreasing part can be the consequence of reduction of food intake, such as a diet to reverse the effects of the initial increase. 

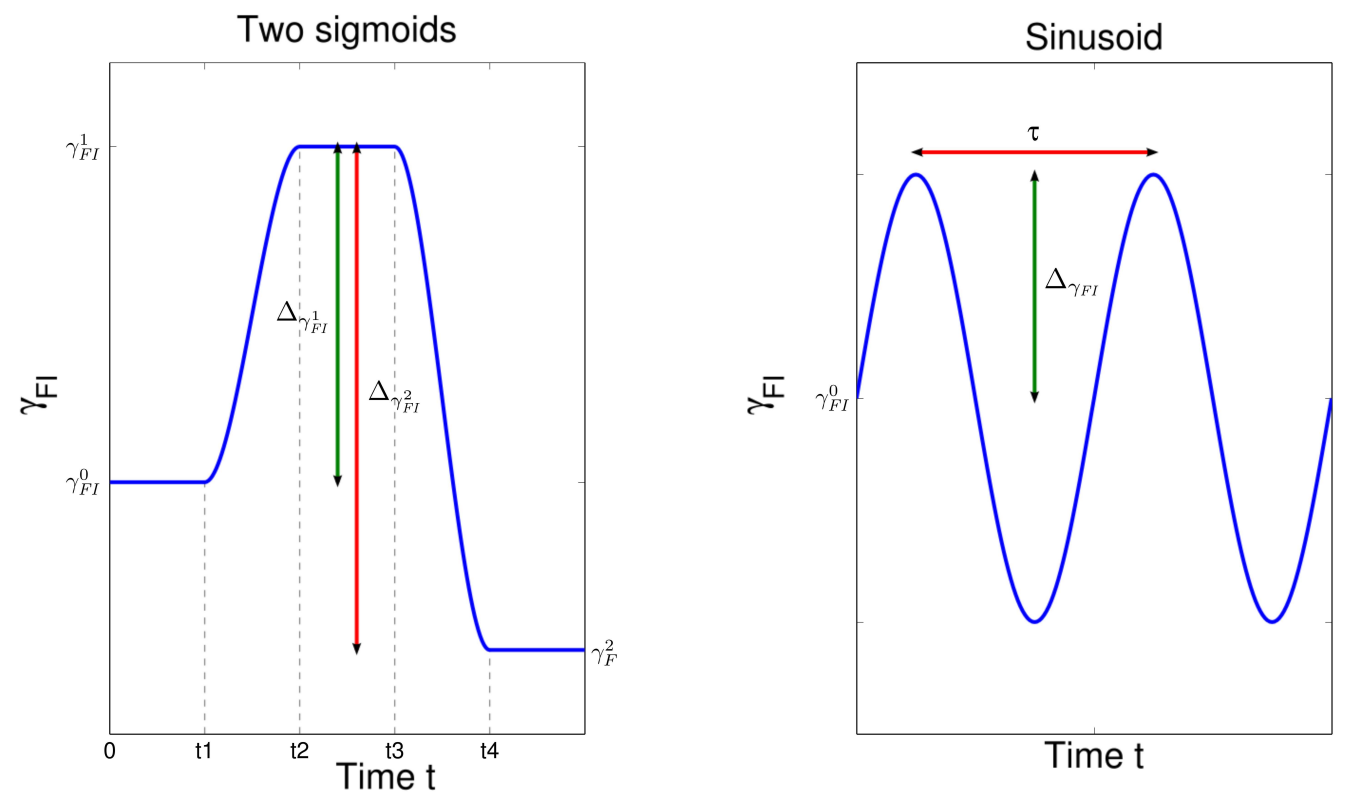

Figure 3: Functions used to temporally modify the stimulation rate of food intake $\left(\gamma_{F I}\right)$ : a double sigmoid (left) and a sinusoid (right).

The sinusoid function is defined by

$$
g(t)=\Delta_{\gamma_{F I}} \sin \left(\frac{2 \pi}{\tau} t\right)
$$

with $\Delta_{\gamma_{F I}}$ representing half the amplitude of variation of the function and $\tau$ the period of the sinusoid. This function describes regular increase and decrease of food intake stimulation with a period of $\tau$ minutes and can model a repetition of the double sigmoid function. It can simulate conscious repeated attempts to limit food intake after an increase. For small periods, it can represent day to day compensations in food intake.

\section{Results}

\subsection{Existence of equilibria and stability analysis}

Equilibria of System (10) correspond to constant solutions. Only the positive solutions are hereafter considered to be physiologically relevant.

We can deduce from System (10) that an equilibrium $\left(F M^{*}, L^{*}, R^{*}, F I^{*}\right)$ satisfies the conditions

$$
\begin{aligned}
L^{*} & =\frac{\gamma_{L}}{\delta_{L}} F M^{*}, \\
R^{*} & =\frac{\gamma_{R}\left(1+\lambda_{R 1} L^{*}\right)}{\delta_{R}\left(1+\lambda_{R 2} L^{* 2}\right)}, \\
F I^{*} & =\frac{\gamma_{F I}\left(L^{* n}+\theta^{n}\right)}{\delta_{F I}\left(L^{* n}\left(1+\phi R^{*}\right)+\theta^{n}\right)},
\end{aligned}
$$

and

$$
F I^{*}=\frac{\eta}{\gamma_{E}}\left(\frac{\rho_{F F M} \gamma_{\Omega} \alpha}{\kappa} \exp \left(\kappa F M^{*}\right)+\left(\rho_{F M}+\rho_{F F M} \gamma_{\Omega}\right) F M^{*}+\rho_{F F M} C+\xi\right) .
$$

Using (13) and (14), the expressions (15) and (16) for $F I^{*}$ can be written as functions of $F M^{*}$. We define

$$
f_{1}(F M):=\frac{\gamma_{F I} \delta_{R}\left(\delta_{L}^{2}+\lambda_{R 2} \gamma_{L}^{2} F M^{2}\right)\left(\gamma_{L}^{n} F M^{n}+\delta_{L}^{n} \theta^{n}\right)}{\delta_{F I}\left(a F M^{n+2}+b F M^{n+1}+c F M^{n}+d F M^{2}+e\right)}
$$


with $a=\gamma_{L}^{n+2} \delta_{R} \lambda_{R 2}, b=\gamma_{L}^{n+1} \phi \delta_{L} \gamma_{R} \lambda_{R 1}, c=\gamma_{L}^{n} \delta_{L}^{2}\left(\delta_{R}+\phi \gamma_{R}\right), d=\delta_{L}^{n} \theta^{n} \delta_{R} \gamma_{L}^{2}$ and $e=\delta_{L}^{n+2} \delta_{R} \theta^{n}$, and

$$
f_{2}(F M):=\frac{\eta}{\gamma_{E}}\left(\frac{\rho_{F F M} \gamma_{\Omega} \alpha}{\kappa} \exp (\kappa F M)+\left(\rho_{F M}+\rho_{F F M} \gamma_{\Omega}\right) F M+\rho_{F F M} C+\xi\right) .
$$

An intersection between $f_{1}$ and $f_{2}$ defines a value $F M^{*}$, and consequently an equilibrium of System (10). Positive equilibria exist if

$$
f_{1}(0)>f_{2}(0),
$$

as $f_{2}(F M)$ is strictly increasing and $f_{1}$ admits an upper bound for $F M=0$. Therefore the system must satisfy the following condition:

$$
\frac{\gamma_{F I}}{\delta_{F I}}>\frac{\eta}{\gamma_{E}}\left(\frac{\rho_{F F M} \gamma_{\Omega} \alpha}{\kappa}+\rho_{F F M} C+\xi\right) .
$$

This condition displays a relationship between food intake (represented by the ratio $\gamma_{F I} / \delta_{F I}$ ) and energy expenditure. In order to obtain positive equilibria, the energy balance $E I-E E$ must be positive for low fat mass (one may note that the same condition ensures positivity of the solutions of System (10), see the end of Section 3.1). The number of equilibria is then equal to one or three depending on the parameter values (see Figure 4 for examples). A detailed analysis of a simplified model is presented in A.

The equilibrium with low leptin concentration and low fat mass (see Figures 4.B and 4.C) corresponds to a healthy state while the equilibrium with high leptin concentration and high fat mass corresponds to an obese state with leptin resistance. In addition, the healthy state has a high number of receptors whereas the obese state has a low number of receptors. One may note that fat free mass is also increased in the obese state, compared to the healthy state, as creation of fat mass leads to creation of some fat free mass, yet it has been observed experimentally that the increase in fat-free mass is smaller than the increase in fat mass [22] and the same is obtained with the set of parameter values in Table 2 .

To study the stability of equilibria, we determine the Jacobian matrix $J$ of System (10) at a given equilibrium $\left(F M^{*}, L^{*}, R^{*}, F I^{*}\right)$, given by

$$
J=\left(\begin{array}{cccc}
J_{L L} & 0 & 0 & J_{M L} \\
J_{L R} & J_{R R} & 0 & 0 \\
J_{L I} & J_{R I} & J_{I I} & 0 \\
0 & 0 & J_{I M} & J_{M M}
\end{array}\right),
$$

with:

$$
\begin{aligned}
J_{L L} & =-\delta_{L}<0, & J_{M L} & =\gamma_{L}>0, \\
J_{R R} & =-\delta_{R}\left(1+\lambda_{R 2} L^{* 2}\right)<0, & J_{L R} & =\gamma_{R} \lambda_{R 1}-2 \delta_{R} \lambda_{R 2} L^{*} R^{*}, \\
J_{I I} & =-\delta_{F I}<0, & J_{L I} & =\frac{-n \gamma_{F I} \theta^{n} \phi L^{* n-1} R^{*}}{\left(L^{* n}\left(1+\phi R^{*}\right)+\theta^{n}\right)^{2}}<0, \\
J_{R I} & =\frac{-\phi \gamma_{F I} L^{* n}\left(L^{* n}+\theta^{n}\right)}{\left(L^{* n}\left(1+\phi R^{*}\right)+\theta^{n}\right)^{2}}<0, & J_{I M} & =\frac{\gamma_{E}}{\rho_{F F M} \gamma_{\Omega}\left(1+\alpha \exp \left(\kappa F M^{*}\right)\right)+\rho_{F M}}>0,
\end{aligned}
$$

and, using (16),

$$
J_{M M}=-\eta<0 .
$$

The only coefficient with a non constant sign is $J_{L R}$, and $J_{L R}$ is positive when

$$
L^{*}<\tilde{L}:=\frac{-\lambda_{R 2}+\sqrt{\lambda_{R 1}^{2} \lambda_{R 2}+\lambda_{R 2}^{2}}}{\lambda_{R 1} \lambda_{R 2}},
$$

or equivalently

$$
F M^{*}<\tilde{F M}:=\frac{\delta_{L}}{\gamma_{L}} \tilde{L} .
$$

The characteristic polynomial $P$ is then defined as

$$
P(\chi)=\chi^{4}+u \chi^{3}+v \chi^{2}+w \chi+z, \quad \chi \in \mathbb{C},
$$



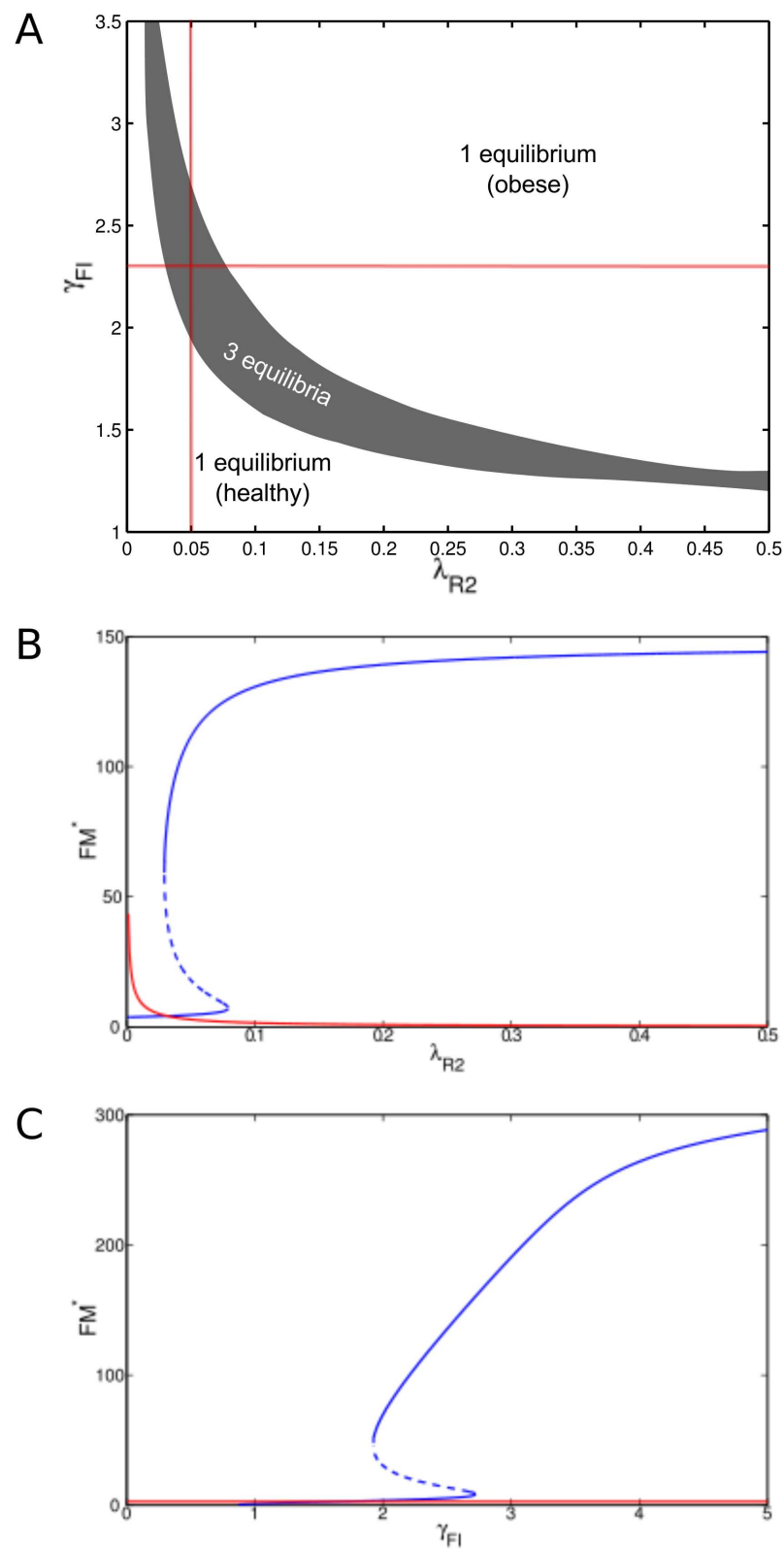

Figure 4: A. Diagram displaying the existence of equilibria in the $\left(\gamma_{F I}, \lambda_{R 2}\right)$-plane (other parameter values are fixed, see Table 2). When increasing parameter values, the system encounters bifurcations with hysteresis. The red lines indicate the sections displayed in B and C. B. Bifurcation diagram for $\lambda_{R 2}$, characterizing the influence of leptin on receptors degradation, with $\gamma_{F I}=2.3$ g. min $^{-1}$ and all other parameter values given by Table 2. C. Bifurcation diagram for $\gamma_{F I}$, representing the stimulation rate of food intake, with $\lambda_{R 2}=0.05 \mathrm{~mL}^{2} . n g^{-2}$ and all other parameter values given by Table 2 . The system displays between one and three equilibria and a hysteresis when increasing the parameter value. Solid blue lines indicate stable equilibria, whereas dashed blue lines indicate unstable equilibria. Stable equilibria fulfill conditions (18), with in particular $z>0$. The value of $\tilde{F M}$, defined in (17), is displayed as a red line. 
with:

$$
\begin{aligned}
u & =-J_{I I}-J_{L L}-J_{R R}-J_{M M}>0, \\
v & =J_{I I} J_{L L}+J_{I I} J_{R R}+J_{I I} J_{M M}+J_{L L} J_{R R}+J_{L L} J_{M M}+J_{R R} J_{M M}>0, \\
w & =-J_{I I} J_{L L} J_{R R}-J_{I I} J_{L L} J_{M M}-J_{I I} J_{R R} J_{M M}-J_{L L} J_{R R} J_{M M}-J_{I M} J_{L I} J_{M L}>0, \\
z & =J_{I I} J_{L L} J_{R R} J_{M M}+J_{I M} J_{L I} J_{R R} J_{M L}-J_{I M} J_{L R} J_{R I} J_{M L} .
\end{aligned}
$$

The sign of $z$ changes depending on parameter and equilibrium values. In particular, if $F M^{*} \leq$ $\tilde{F M}$, then both $J_{L R}$ and $z$ are positive.

If real parts of the roots of the characteristic polynomial are negative, the associated equilibrium is locally asymptotically stable while if at least one root has a positive real part, the equilibrium is unstable.

The Routh-Hurwith Criterion, applied to $P$, allows us to conclude that all the roots of $P(\chi)$ are negative or have a negative real part if and only if the following conditions are satisfied,

$$
\left\{\begin{aligned}
z & >0 \\
u v & >w \\
w(u v-w) & >u^{2} z .
\end{aligned}\right.
$$

For a given set of parameter values, the system displays 3 equilibria, an unstable one between 2 stable ones, with a hysteresis (see Figure 4.A and A for a detailed stability analysis of a simplified system). It is easy to numerically determine the equilibria values and the area of stability (see Figures 4.B and 4.C for examples of $\lambda_{R 2}$ and $\gamma_{F I}$ dependent stability areas). With the parameter values used for Figures 4.B and 4.C, conditions $u v>w$ and $w(u v-w)>u^{2} z$ are satisfied as soon as $z>0$, and the stability is consequently determined only by the sign of $z$. In particular, for values of $F M^{*}$ lower than $F M, z$ is positive and the equilibrium is stable.

It must be noted that, for instance, when $\lambda_{R 1}=0$ (absence of positive regulation of leptin receptor production by leptin), bistability also occurs, event though $J_{L R}<0$ : the condition $J_{L R}>0$ is not a necessary and sufficient condition for bistability, because $z$ can be positive even though $J_{L R}$ is negative.

System (10) can then be bistable or monostable, in this latter case it can either be stable around a healthy or an obese equilibrium. The development of leptin resistance and obesity can only occur if the solution reaches the basin of attraction of the obese equilibrium. Dynamically going from one equilibrium to the other one can only be achieved by perturbing the parameter values influencing the existence of equilibria and the size of the basins of attraction.

\subsection{Constant leptin infusion can lead to leptin resistance}

Leptin resistance is characterized by the inability of the system to integrate leptin signals. Constant injections of leptin in healthy rats showed that after an initial phase of efficient regulation of food intake a second phase, occurring after a few days, corresponding to the development of leptin resistance, was associated with high food intake and high leptin levels [35, 37].

We first assume that body weight remains constant, with both fat mass and fat-free mass constant, and we model a constant injection of leptin. Let denote by $\Lambda\left(n g \cdot m L^{-1} \cdot \mathrm{min}^{-1}\right)$ the leptin injection, then Equation (6) can be written

$$
\frac{\mathrm{d} L}{\mathrm{~d} t}=\gamma_{L} F M+\Lambda-\delta_{L} L
$$

with $F M$ constant.

Starting close to the healthy equilibrium (for variables $L, R$ and $F I$, whereas $F M$ is constant), we observe in Figure 5:

- first, an increase in leptin level, an increase in the density of receptors, and a decrease in food intake, corresponding to a healthy behavior;

- then, a higher leptin level, a decrease of the density of leptin receptors to low levels, and an increase in food intake which stabilizes at a value higher than the initial value. 

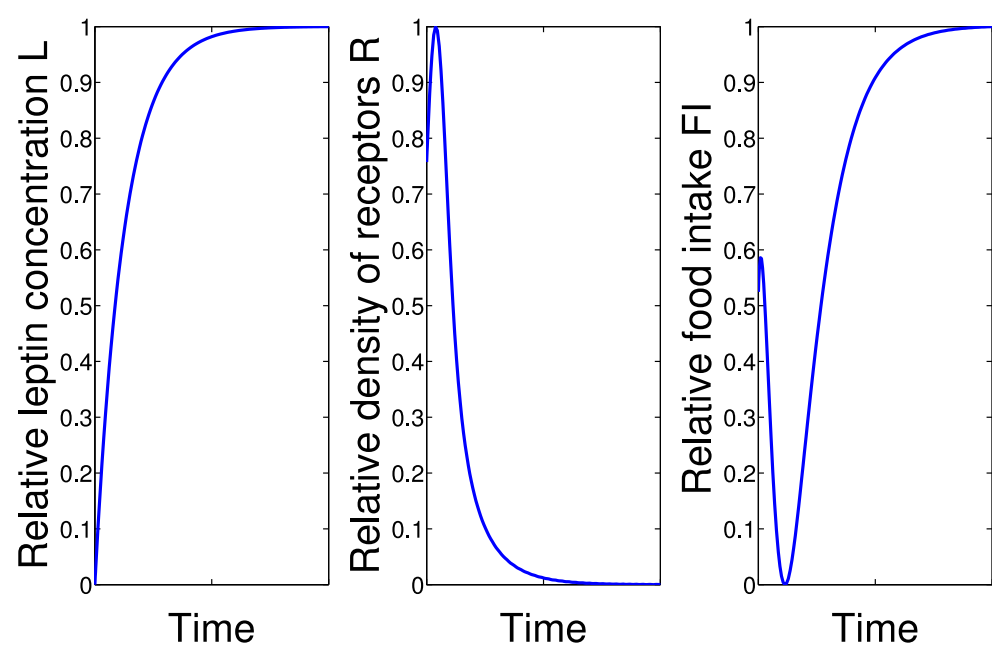

Figure 5: Relative values of leptin, leptin receptors and food intake (normalized between 0 and 1 , for illustration purpose, using the following formula: $(x(t)-\min (x(t))) /(\max (x(t))-\min (x(t)))$, where $x=R, L, F I)$, following a constant leptin injection. The initial value is close to the healthy equilibrium.

This situation is characteristic of leptin resistance. Depending on the intensity of the injection, results quantitatively change, yet they are qualitatively equivalent.

If the starting point is close to the obese equilibrium, the system is already leptin resistant. Leptin injection in that case will have no impact on the dynamics of the system (results not shown). Also in this case, depending on the initial value and the strength of the injection, one can observe a slight decrease in the density of receptors and a slight increase in food intake: the system is becoming more leptin resistant.

We now use the full model (System (10)) with leptin dynamics given by (19) to illustrate the appearance of leptin resistance. We use experimental data from Pal and Sahu [35], describing food intake and body weight dynamics in rats during a constant injection of leptin. Results are presented in Figure 6. In addition to simulation results for food intake and body weight dynamics (Figures 6.A and 6.B) we also present simulated leptin dynamics and leptin receptor dynamics (Figure 6.C and 6.D). The constant leptin injection starts at day 0, and previously the system is in a healthy state. Following leptin injection one observes a strong decrease in food intake which increases again after 3-4 days, and a slower decrease of body weight which increases again from day 10. The model correctly reproduces these dynamics (Figures 6.A and 6.B). In addition, due to a constant leptin injection from day 0 the leptin concentration quickly reaches a plateau and saturates throughout the experiment, while the number of leptin receptors continuously decreases towards low levels (Figures 6.C and 6.D). This situation characterizes leptin resistance.

\subsection{Varying the stimulation rate of food intake can induce leptin resis- tance and obesity}

We showed in the previous section that our model is able to characterize the development of leptin resistance. We are now going to theoretically investigate its ability to describe pathways to leptin resistance and obesity in order to make predictions that would be testable experimentally.

We are particularly interested in the influence of progressive variations in food intake on the development of leptin resistance and obesity. Leptin resistance is characterized by a high concentration of leptin $L$ which is not associated with a decrease in fat mass $F M$. Obesity corresponds in this model to a state of the system with increased fat mass $F M$. Variations in food intake are indeed influenced by variations in the stimulation rate of food intake, represented by the parameter $\gamma_{F I}$ in $(9)$. Variations in the rate of inhibition $\left(\delta_{F I}\right)$ can be realistically neglected. 
A

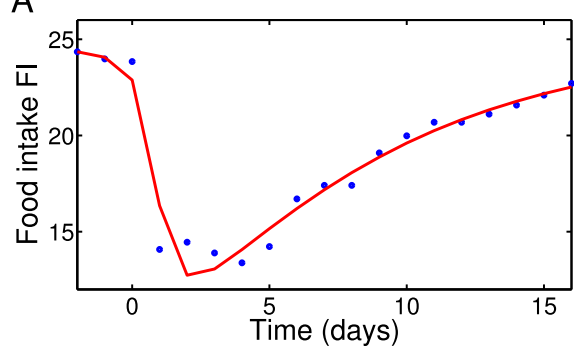

C

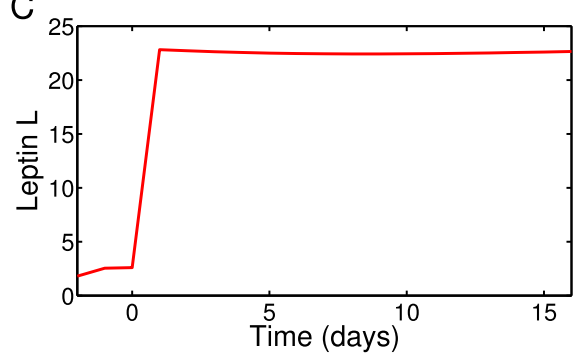

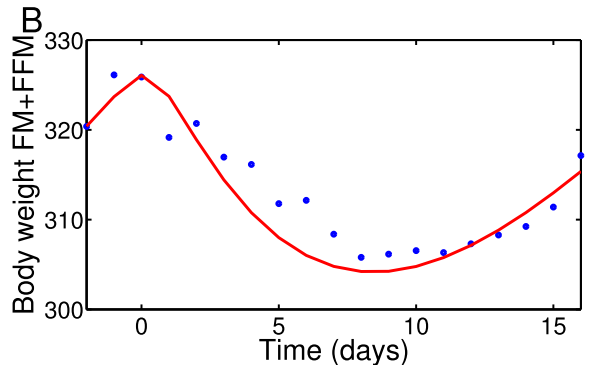

$\mathrm{D}$

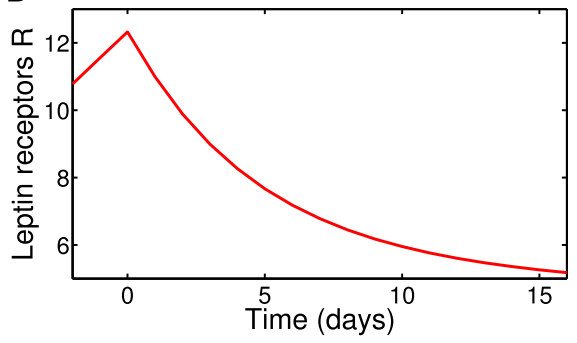

Figure 6: Simulation results (red lines) compared to [35] data (blue dots representing the mean value at each time). Experimental data have been obtained by infusing leptin for 16 days directly into the brain of Sprague-Dawley rats. Constant injection of leptin starts at time 0 and lasts until the end of the experiment (day 16). Parameter values are taken from Jacquier et al. [25] or estimated (see Table 3). A. Food intake dynamics. Food intake drops at day 0, then stays low for a few days and increases to its initial level. B. Body weight dynamics. Body weight starts to decrease when leptin injection starts and then increases from day 10. C. Leptin concentration. It becomes and remains high following the injection, totally saturating leptin receptors and inducing a downregulation of the receptors. D. Leptin receptors. They increase previous to the leptin injection, then continuously decrease until the end of the experiment. The system is progressively becoming leptin resistant. 


\begin{tabular}{|c|c|c|c|}
\hline Parameter & Unit & Value & Reference \\
\hline$\gamma_{L}$ & $\overline{n n g \cdot g^{-1} \cdot \mathrm{min}^{-1}}$ & 0.0954 & derived \\
\hline$\delta_{L}$ & min $^{-1}$ & 0.074 & [48] \\
\hline$\gamma_{R}$ & mol. $L^{-1} \cdot$ min $^{-1}$ & $5.87 \times 10^{-4}$ & derived \\
\hline$\delta_{R}$ & $\min ^{-1}$ & $3.26 \times 10^{-6}$ & derived \\
\hline$\lambda_{R 1}$ & $n g^{-1}$ & $1.8 \times 10^{-4}$ & derived \\
\hline$\lambda_{R 2}$ & $n g^{-2}$ & $1.94 \times 10^{-4}$ & derived \\
\hline$\delta_{F I}$ & $\min ^{-1}$ & $1.19 \times 10^{-3}$ & derived \\
\hline$\gamma_{F I}$ & kcal.min $^{-1}$ & $3.46 \times 10^{-4}$ & derived \\
\hline$\phi$ & L.mol ${ }^{-1}$ & 1 & derived \\
\hline$\theta$ & $n g \cdot m L^{-1}$ & 57.22 & derived \\
\hline$n$ & N.U. & 2 & derived \\
\hline$\gamma_{\Omega}$ & N.U. & 2.2 & [25] \\
\hline$\alpha$ & N.U. & $7.27 \times 10^{-10}$ & [25] \\
\hline$\kappa$ & $g^{-1}$ & 0.269 & [25] \\
\hline$\gamma_{E}$ & min $^{-1}$ & 1 & derived \\
\hline$\eta$ & min $^{-1}$ & $1.77 \times 10^{-5}$ & derived \\
\hline$\rho_{F F M}$ & kcal.g $g^{-1}$ & 9.4 & {$[18,19]$} \\
\hline$\rho_{F M}$ & kcal.g $g^{-1}$ & 1.8 & {$[18,19]$} \\
\hline$\xi$ & $k c a l$ & 1413.6 & derived \\
\hline$\Lambda$ & ng.min ${ }^{-1}$ & 30 & derived \\
\hline
\end{tabular}

Table 3: Parameter units and values used to generate simulated dynamics from System (10) compared with data from [35], and presented in Figure 6. N.U. denotes "non-dimensional unit", when the value is taken from the literature, the corresponding reference is indicated.

We consider 2 scenarii corresponding to 2 different ways of varying food intake. The parameter $\gamma_{F I}$ is assumed to be given by

$$
\gamma_{F I}=\gamma_{F I}(t)=\gamma_{F I}^{0}+g(t),
$$

where $\gamma_{F I}^{0}$ represents a basal value of food intake stimulation (which depends on individual characteristics, so variations in $\gamma_{F I}^{0}$ account for inter-individual variability), and $g(t)$ describes a timedependent modification of food intake habits. We consider either increasing then decreasing (double sigmoid) variations or oscillating (sinusoidal) variations (see Figure 3 and Section 2.2). Variations in the value of $\gamma_{F I}$ induce modifications in equilibria values and stability. In the bistable case, a solution of System (10) cannot go from one equilibrium to the other, so the only way to develop leptin resistance and obesity starting from a healthy state is that, due to some perturbation, the healthy stable equilibrium no longer exists at some point.

\subsubsection{Increasing then decreasing food intake stimulation rate}

We study the effect of progressive changes in food intake on the development of leptin resistance and obesity. These changes are represented by an increase of the stimulation rate $\gamma_{F I}$, followed by a stabilization and later a decrease. We assume that $\gamma_{F I}^{0}$ corresponds either to the bistable system or the system with only the monostable healthy equilibrium and that the initial condition of System (10) is close to the healthy equilibrium.

The initial increase between $\gamma_{F I}^{0}$ and $\gamma_{F I}^{1}=\gamma_{F I}^{0}+\Delta_{\gamma_{F I}^{1}}$ leads to an increase in body weight. The importance of the increase depends on the amplitude $\Delta_{\gamma_{F I}^{1}}$ and the duration $t_{2}-t_{1}$ (see (11)). If $\gamma_{F I}^{1}$ is in the bistable area, the solution remains close to the healthy equilibrium. Then the increase in fat mass is limited to normal physiological variations, without development of leptin resistance and obesity. Yet, with a plateau value $\gamma_{F I}^{1}$ corresponding to a monostable obese equilibrium the fat mass keeps increasing and the solution reaches the basin of attraction of the obese equilibrium, characterized by high fat mass and leptin levels (see Figure 7.A). This represents a progressive pathway to leptin resistance and obesity, where a progressive increase in food consumption has 
almost no impact for some time but can lead to leptin resistance and obesity if the increase does not stop.
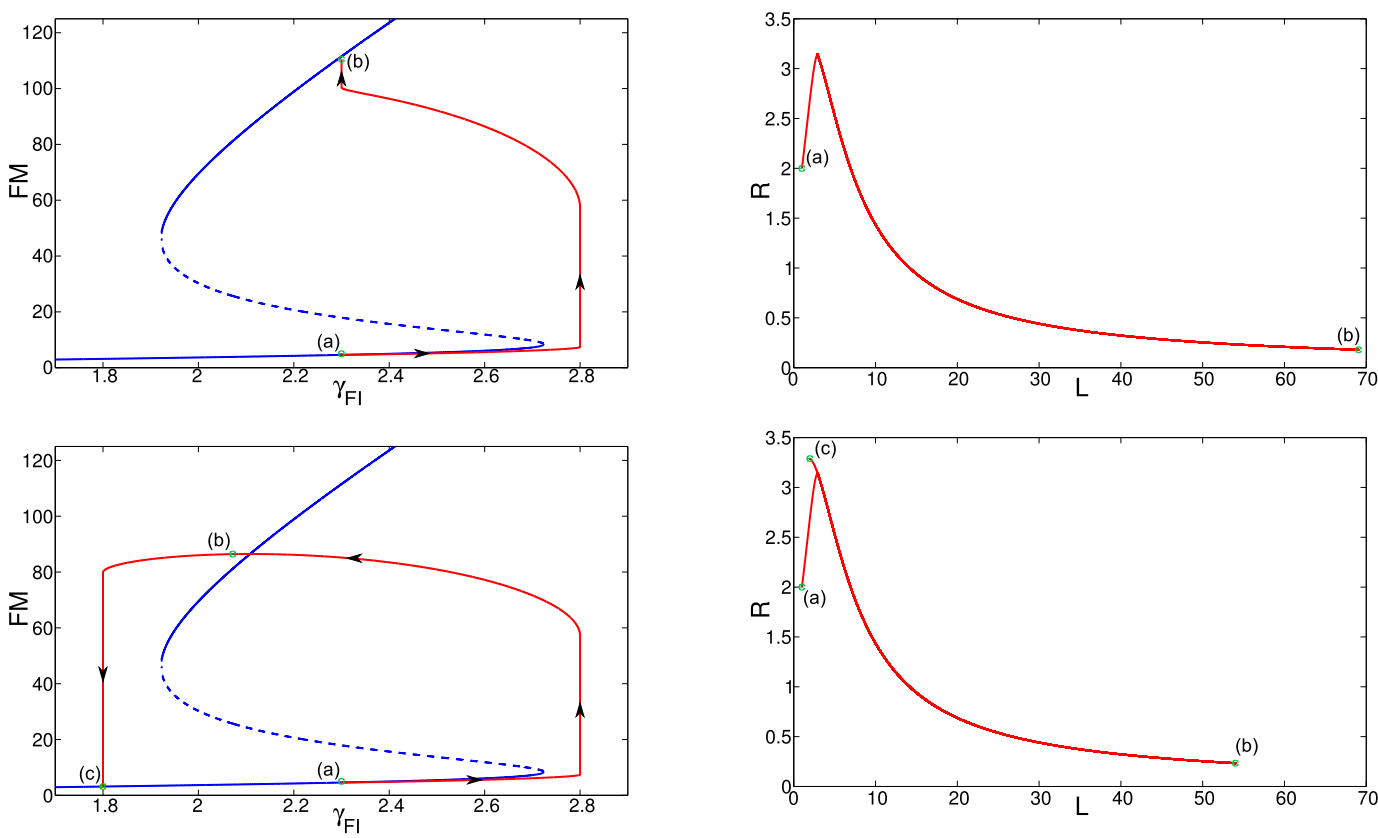

Figure 7: Evolution of the value of $F M$ (in red) with the values of the equilibrium $F M^{*}$ (in blue) on the left column, and evolution of the values of $L$ and $R$ on the right column, for an increasing then decreasing sigmoid-like function $\gamma_{F I}$. The initial condition of System (10), denoted by (a), is close to the healthy equilibrium and $\gamma_{F I}^{0}$ is located in the bistable area. A. For $\gamma_{F I}$ increasing from 2.3 to 2.8 and then going back to 2.3 , the solution goes from the healthy equilibrium (a) to the obese equilibrium (b). The system progressively becomes leptin resistant, with low density of receptors and high concentration of leptin at the end of the variation. B. For $\gamma_{F I}$ increasing from 2.3 to 2.8 and then decreasing to 1.8 , the solution reaches the obese equilibrium before going back to the healthy equilibrium. The system, initially healthy (a), becomes leptin resistant (b), and then returns to the healthy state (c) when $\gamma_{F I}$ reaches its final value. This evolution follows an hysteresis cycle.

The same variation characterized by $\Delta_{\gamma_{F I}^{1}}$ and $\Delta_{\gamma_{F I}^{2}}$ has different consequences depending on the initial value $\gamma_{F I}^{0}$ (see Figure 8). Thus, a fixed or $\gamma_{F I}^{0}$-dependent variation will lead to different values of the system after some time. Depending on the value $\gamma_{F I}^{0}$, the system either stays in the healthy state or becomes obese and leptin resistant. Indeed inter-individual variability has an important impact on the development of leptin resistance and obesity.

Assuming that $\gamma_{F I}^{1}$ is located in the monostable obese area and $\gamma_{F I}^{0}$ in the bistable area, we study the effect of a decrease, occurring after the plateau phase, of the stimulation rate $\gamma_{F I}$ on the solution. The behavior of the solution depends on the amplitude $\Delta_{\gamma_{F I}^{2}}$ of the decrease. If $\Delta_{\gamma_{F I}^{2}} \leq \Delta_{\gamma_{F I}^{1}}$, corresponding to a final stimulation rate $\gamma_{F I}^{2}$ higher or equal to the initial value, the solution remains in the basin of attraction of the obese equilibrium (see Figure 7.A). This situation corresponds to the case where an individual progressively increases their food intake, then becomes leptin resistant and obese but cannot go back to their initial state (healthy) even by reducing food intake to the original level. One may note that this situation can also occur for $\Delta_{\gamma_{F I}^{2}}>\Delta_{\gamma_{F I}^{1}}$, if $\gamma_{F I}^{2}$ corresponds to the bistable area.

In order for the solution to go back to the healthy equilibrium, the decrease in the stimulation rate must be more important than the increase and the final value $\gamma_{F I}^{2}$ must correspond to the monostable healthy equilibrium. The solution then follows an hysteresis cycle when increasing and then decreasing (see Figure 7.B). In order to return to the healthy state from the obese state, the 


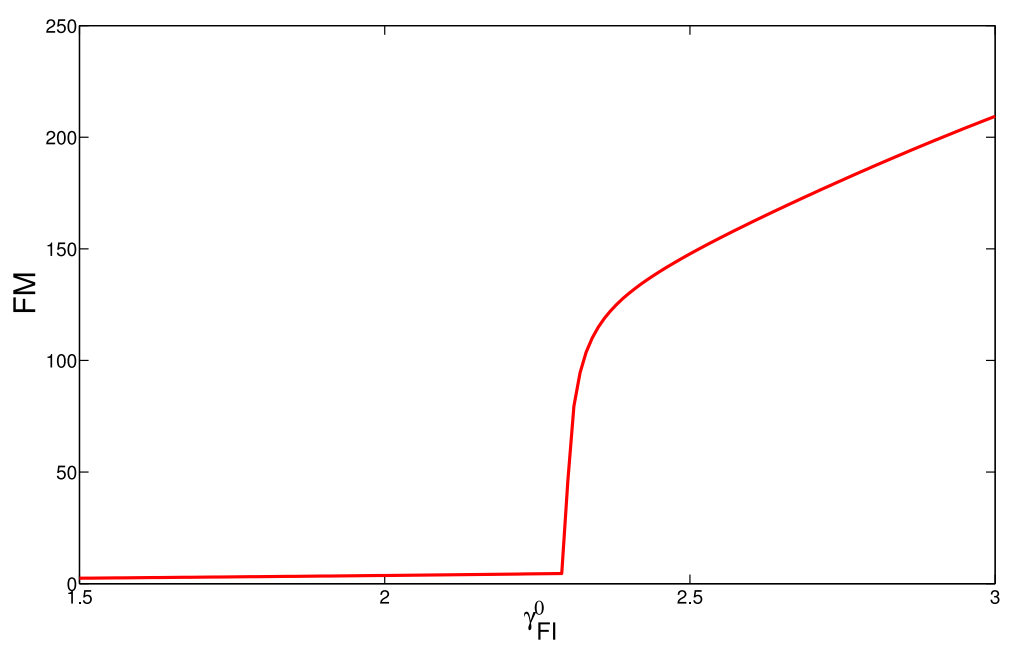

Figure 8: Final value of the fat mass $F M$ after an increase of the food intake stimulation $\gamma_{F I}$, followed by a decrease (sigmoid-like function, see Figure 3 left). The initial condition of System (10) is close to the healthy equilibrium. The initial value of $\gamma_{F I}^{0}$ ranges from 1.5 to 3 and the amplitude of the variation depends on the initial value $\left(\Delta_{\gamma_{F I}^{1}}=\Delta_{\gamma_{F I}^{2}}=0.2 \gamma_{F I}^{0}\right)$.

food intake stimulation must be sustained at a lower value than it was at the beginning, for a time long enough.

One may note that if the plateau time (between $t_{2}$ and $t_{3}$ ) is too short, the solution may not reach the basin of attraction of the obese equilibrium and just varies around the healthy equilibrium. Short-time increases of food intake stimulation rate do not significantly impact body weight and can be easily compensated, which is not the case for a sustained increase.

\subsubsection{Oscillating food intake stimulation}

We study the impact of repeated increases and decreases in food intake stimulation on the development of leptin resistance and obesity. These variations are modeled as a sine function centered on $\gamma_{F I}^{0}$, as described in (12). We assume that $\gamma_{F I}^{0}$ corresponds either to the bistable system or the system with only the monostable healthy equilibrium, and that the initial condition of System (10) is close to the healthy equilibrium. One may note that varying $\gamma_{F I}$ to follow a sine function leads to oscillations in variable values at the same frequency as $\gamma_{F I}(t)$. Moreover, depending on the initial value $\gamma_{F I}^{0}$, the amplitude and the period of oscillations, the behavior of the system will follow different patterns. In the following, we describe these different cases.

We first consider the case with $\gamma_{F I}$ varying only in the bistable area. The solution oscillates around the equilibrium value closer to the initial condition of System (10) (obese or healthy, results not shown). This can represent day to day variations observed in most biological systems and that have almost no impact on the long-term body weight.

We assume now that the variations of $\gamma_{F I}$ cover the entire bistable area and parts of the monostable areas both on the left and right sides of the bistable area. We also assume that $\gamma_{F I}^{0}$ is located close to the center of the bistable area, in order to have the same time spent in both monostable areas. Though, the solution should have the same possibility to join the basin of attraction of the remaining equilibrium in both monostable areas. The period of oscillations also has an impact on the behavior of the system. For example, the amplitude of the variation in fat mass is a function of the period of the oscillations (see Figure 9.A). If the period of oscillations is low, the solution oscillates around the healthy equilibrium, even if, for some values of $\gamma_{F I}$, the healthy equilibrium does not exist anymore (see Figure 9.B). As the period of oscillations is low, the oscillations can model short term variations in food intake, that have only a limited impact on body weight dynamics [7]. Increasing the period of oscillations leads to changes in the dynamics 
A
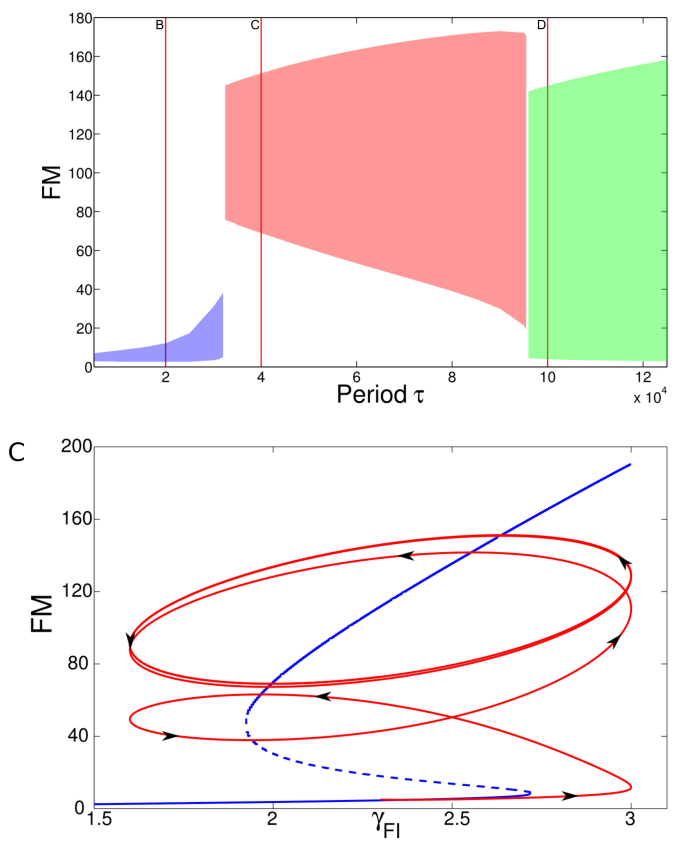

B
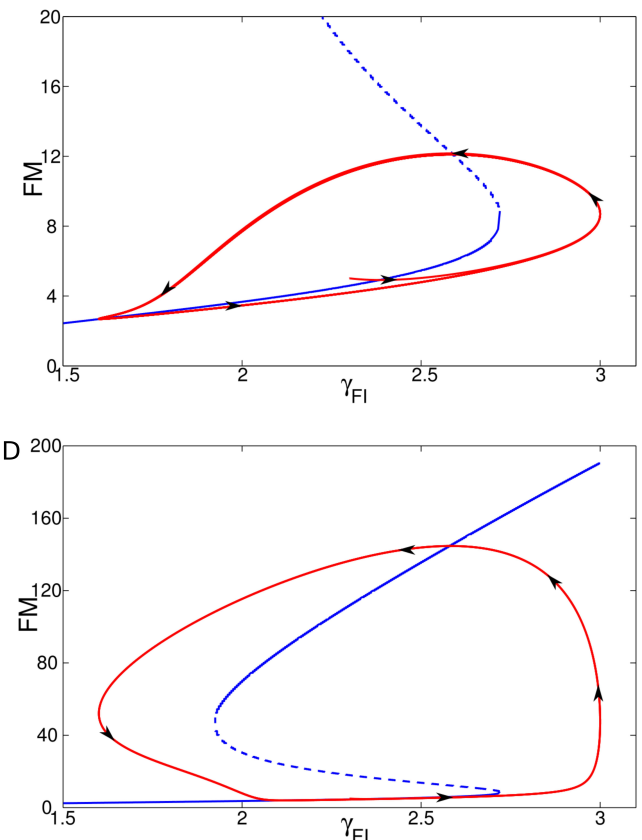

Figure 9: Evolution of fat mass $F M$ when the stimulation rate of food intake $\gamma_{F I}$ oscillates between 1.6 and 3 as a sine function. The initial condition of System (10) is close to the healthy equilibrium and $\gamma_{F I}^{0}=2.3$. A. Evolution of the amplitude of the sustained oscillations of $F M$ (colored areas) for $\gamma_{F I}$ oscillating with an increasing period $\tau$. The amplitude $\Delta_{\gamma_{F I}}$ remains the same. Three areas corresponding to different amplitudes of $F M$ are observed: for a low period $\tau$ (in blue), for an intermediate period (in red) and for a high period (in green). The period of oscillations corresponding to figures B, C and D is displayed by vertical red lines. B-C-D. Evolution of the value of $F M$ (in red) and of the value of the equilibrium $F M^{*}$ (in blue) as a function of $\gamma_{F I}$. B. For a low period of oscillations, the solution oscillates around the healthy equilibrium. The system is not leptin resistant (low leptin level, high density of receptors). C. The period of oscillations is doubled and the solution oscillates around the obese equilibrium. The solution reaches a limit cycle where the system is in a leptin resistant state (low density of receptors and high concentration of leptin). D. For a period of oscillations equal to 5 times the period of Figure A, the solution oscillates between the healthy and the obese equilibrium. The system oscillates between a state of leptin resistance and a healthy state.

of the solution, which can leave the healthy equilibrium to oscillate around the obese equilibrium (see Figure 9.C). In that case, the body weight is trapped around the obese state and it is not possible for the individual to leave the obese state, despite a periodic reduction in food intake. The individual is also leptin resistant, with a low density of receptors and a high concentration of leptin. If the period of oscillations is large enough, the solution oscillates between the two stable equilibria (see Figure 9.D). This latter case could correspond to a yo-yo effect observed in individuals who progressively gain weight before following a strict diet and repeat the process several times. One may notice that increasing the amplitude of oscillations eases the change of equilibrium: the period of oscillations needed to leave the healthy equilibrium is reduced. From a biological point of view, if a healthy individual increases their food intake in an important way, it should be easier and quicker to reach an obese state with leptin resistance than slightly increasing food intake. A similar phenomenon is also observed when going from the obese state to the healthy state by decreasing food intake.

If $\gamma_{F I}$ ranges in the bistable area and in the monostable obese equilibrium area only, the behavior of the solution is similar to the previous case (results not shown) except that it is not possible to go back to the healthy equilibrium. For a given amplitude of oscillations and an initial condition 
for the System (10) close to the healthy equilibrium, a low period of oscillations corresponds to oscillations around the healthy equilibrium. If the period of oscillations is increased, the solution can reach the basin of attraction of the obese equilibrium when the system is monostable and then oscillates around the obese equilibrium.

When the solution oscillates around the obese equilibrium without being able to go back to the healthy equilibrium (see for instance Figure 9.C), the only possibility to go back to the healthy state from the obese state is to apply a different perturbation to the system. This perturbation can be applied to any parameter of the system and should allow the system to reach the monostable healthy area for a time long enough for the solution to join and remain in the basin of attraction of the healthy equilibrium.

\section{Discussion}

Leptin resistance is observed in humans and in rodents, and is characterized by the inability of the body to respond to high concentrations of leptin in the blood, which should normally induce a downregulation of food intake. Mechanisms behind the development of this resistance are not fully known. Obesity, associated with high amounts of fat and leptin in the body, is a cause and a consequence of leptin resistance [50].

In this work, we developed a mathematical model of body weight and food intake dynamics, considering a regulation mediated only by the leptin/leptin receptors system. It is noticeable that regulatory mechanisms have been inspired by experimental observations in rodents and previous models of body weight dynamics for rodents. Although describing a simplified reality, this system has 2 stable equilibria (depending of course on parameter values) associated to a healthy state (no leptin resistance and low fat mass) and a leptin-resistant/obese state (high fat mass and high leptin levels). At a constant healthy fat mass, a constant leptin infusion induces a state of leptin resistance, characterized by an increased leptin concentration, a reduced density of receptors and an increased food intake. We showed that our model was able to correctly reproduce the dynamics of body weight and food intake during leptin injection that leads to development of leptin resistance, using data from Pal and Sahu [35]. We then showed that the system can dynamically go from the healthy state to the leptin resistant one, and described potential pathways to obesity. The underlying mechanism relies on leptin's up and down regulation of its own receptors. High leptin concentration strongly down regulates leptin receptors - by increasing degradation rate - whereas low leptin concentration has the opposite effect. Under this assumption, we showed that it is possible to become leptin resistant and obese, starting from a healthy state, by progressively increasing food intake stimulation rate in order to ignore leptin signals.

We also investigated the potential consequences of a sinusoidal variation of food intake stimulation and showed that it could theoretically lead to leptin resistance and obesity under some conditions on the period and the amplitude of oscillations. Thus, low amplitude and low period oscillations have no impact on the transition from a healthy state to a leptin resistant state. Increasing the period and/or the amplitude of the oscillation increases the probability for the system to become leptin resistant and obese. The extreme case with high amplitude and high period leads to an alternation of the system between healthiness and leptin resistance, which is considered to be totally reversible in our model. This behavior is qualitatively in agreement with the biology, since leptin resistance is considered to be reversible or at least partially reversible. Introducing variability in the initial parameter values leads to different behaviors of the system, which has a different susceptibility to develop leptin resistance and obesity when submitted to the same perturbation. If the food intake stimulation is high, the probability to develop leptin resistance and obesity after a perturbation is more important than for a low value. To our knowledge, the hysteresis cycle obtained when varying parameters values has not been observed experimentally. It may be possible to observe it by monitoring body weight, food intake, leptin concentration and leptin receptors expression when progressively changing the caloric content of food intake over a long time scale in rodents.

One may think about other ways of inducing leptin resistance and obesity. Instead of associating the development of leptin resistance to a temporal modification of one or several parameter values (here, the food intake stimulation rate) a stochastic modification of food intake could lead to leptin 
resistance. Our attempts to induce leptin resistance and obesity by adding a Wiener process to Equation (9), describing food intake dynamics, did not provide relevant results (results not shown): only large amounts of noise were shown to induce a modification of the system, switching from a healthy state to a leptin-resistant state, and they do not appear biologically realistic. It would be more reasonable to consider that stochastic events combined with a temporal modification of some characteristics of the system (as described above) could lead to leptin resistance, consequently they could not be considered as the main cause of leptin resistance.

Another hypothesis for leptin resistance development could be a delay in the integration of leptin signals. For instance, production and/or degradation of leptin receptors may not be instantaneously modified by leptin levels, or food intake stimulation may not react to the current state of the leptin/leptin receptors system. We tested the assumption of a delayed response of food intake regulation to changes in receptor density, yet this does not allow to describe the development of leptin resistance (results not shown): adding a delay in System (10) can destabilize either one or both equilibria, yet the destabilization is associated with the appearance of oscillating solutions, but not with a pathway to leptin resistance. At this stage, a preliminary conclusion would be that a delay can strengthen a leptin resistant situation, but cannot induce leptin resistance.

It is noticeable that our model only includes one type of leptin receptors, located in the hypothalamus, that induce a regulation of food intake. There exist experimental evidences that leptin resistance can also occur at the blood-brain barrier, leading to a reduced ratio between blood leptin and plasma leptin and to an inability of the system to respond to intravenous injections of leptin. It is possible to improve the model by including the transport of leptin from the blood to the brain via its receptors and observe different types of leptin resistance. This is left for a future work, as experimental measurements would be needed to better characterize leptin resistances. It may also be mentioned that other regulators of food intake and body weight exist [29, 30, 40], such as adaptation of the energy expenditure and the effect of hormones other than leptin, which were not considered here. However, the behavior of our model is qualitatively relevant from a biological point of view. This model, consisting in only 4 differential equations, efficiently describes the mechanisms of leptin resistance (without assuming degradations due to aging), at least in rodents, and the development of obesity based on the regulation of leptin receptors density by leptin.

\section{Acknowledgments}

The authors would like to thank Dr C.O Soulage for fruitful discussions, his valuable comments and help when designing the model.

\section{References}

[1] Margaret B Allison and Martin G Myers. 20 years of leptin: Connecting leptin signaling to biological function. Journal of endocrinology, 223(1):T25-T35, Oct 2014.

[2] A. S. Banks, S. M. Davis, S. H. Bates, and M. G. Myers. Activation of downstream signals by the long form of the leptin receptor. The Journal of Biological Chemistry, 275(19):1456314572, May 2000.

[3] W. A. Banks, C. R. DiPalma, and C. L. Farrell. Impaired transport of leptin across the blood-brain barrier in obesity. Peptides, 20(11):1341-1345, Nov 1999.

[4] C. Bjørbaek, S. Uotani, B. da Silva, and J. S. Flier. Divergent signaling capacities of the long and short isoforms of the leptin receptor. The Journal of Biological Chemistry, 272(51):3268632695, Dec 1997.

[5] J. F. Caro, J. W. Kolaczynski, M. R. Nyce, J. P. Ohannesian, I. Opentanova, W. H. Goldman, R. B. Lynn, P. L. Zhang, M. K. Sinha, and R. V. Considine. Decreased cerebrospinalfluid/serum leptin ratio in obesity: a possible mechanism for leptin resistance. Lancet, 348(9021):159-161, Jul 1996.

[6] Carson C Chow and Kevin D Hall. The dynamics of human body weight change. PLoS Computational Biology, 4(3):e1000045, Mar 2008. 
[7] Carson C Chow and Kevin D Hall. Short and long-term energy intake patterns and their implications for human body weight regulation. Physiology \& Behavior, 134:60-65, Jul 2014.

[8] Carmen Sobrino Crespo, Aránzazu Perianes Cachero, Lilian Puebla Jiménez, Vicente Barrios, and Eduardo Arilla Ferreiro. Peptides and food intake. Frontiers in endocrinology, 5:58, 2014.

[9] Albert A de Graaf, Andreas P Freidig, Baukje De Roos, Neema Jamshidi, Matthias Heinemann, Johan A C Rullmann, Kevin D Hall, Martin Adiels, and Ben van Ommen. Nutritional systems biology modeling: from molecular mechanisms to physiology. PLoS Computational Biology, 5(11):e1000554, Nov 2009.

[10] F. A. Duca and M. Covasa. Current and emerging concepts on the role of peripheral signals in the control of food intake and development of obesity. British Journal of Nutrition, 108(5):778793, Sep 2012.

[11] K. El-Haschimi, D. D. Pierroz, S. M. Hileman, C. Bjørbaek, and J. S. Flier. Two defects contribute to hypothalamic leptin resistance in mice with diet-induced obesity. The journal of clinical investigation, 105(12):1827-1832, Jun 2000.

[12] J. M. Friedman. Leptin, leptin receptors, and the control of body weight. Nutrition reviews, 56(2 Pt 2):s38-46; discussion s54-75, Feb 1998.

[13] J. M. Friedman. Obesity in the new millennium. Nature, 404(6778):632-634, Apr 2000.

[14] J. M. Friedman and J. L. Halaas. Leptin and the regulation of body weight in mammals. Nature, 395(6704):763-770, Oct 1998.

[15] Jeffrey Friedman. 20 years of leptin: Leptin at 20: an overview. Journal of endocrinology, 223(1):T1-T8, Oct 2014.

[16] Andrea De Gaetano, Thomas Hardy, Benoit Beck, Eyas Abu-Raddad, Pasquale Palumbo, Juliana Bue-Valleskey, and Niels Pørksen. Mathematical models of diabetes progression. American Journal of Physiology - Endocrinology and Metabolism, 295(6):E1462-E1479, Dec 2008.

[17] P. L. Golden, T. J. Maccagnan, and W. M. Pardridge. Human blood-brain barrier leptin receptor. Binding and endocytosis in isolated human brain microvessels. The journal of clinical investigation, 99(1):14-18, Jan 1997.

[18] Juen Guo and Kevin D Hall. Estimating the continuous-time dynamics of energy and fat metabolism in mice. PLoS Computational Biology, 5(9):e1000511, Sep 2009.

[19] Juen Guo and Kevin D Hall. Predicting changes of body weight, body fat, energy expenditure and metabolic fuel selection in C57BL/6 mice. PLoS One, 6(1):e15961, 2011.

[20] Stephan J Guyenet and Michael W Schwartz. Regulation of food intake, energy balance, and body fat mass: implications for the pathogenesis and treatment of obesity. The Journal of Clinical Endocrinology \& Metabolism, 97(3):745-755, Mar 2012.

[21] J. L. Halaas, C. Boozer, J. Blair-West, N. Fidahusein, D. A. Denton, and J. M. Friedman. Physiological response to long-term peripheral and central leptin infusion in lean and obese mice. Proceedings of the National Academy of Sciences, 94(16):8878-8883, Aug 1997.

[22] Kevin D Hall. Body fat and fat-free mass inter-relationships: Forbes's theory revisited. British Journal of Nutrition, 97(6):1059-1063, Jun 2007.

[23] Kevin D Hall. Mathematical modelling of energy expenditure during tissue deposition. British Journal of Nutrition, 104(1):4-7, Jul 2010.

[24] G. W. Horgan. The behaviour of a neutral model of weight regulated only by body mass. Journal of Theoretical Biology, 270(1):1-6, Feb 2011.

[25] Marine Jacquier, Fabien Crauste, Christophe O Soulage, and Hédi A Soula. A predictive model of the dynamics of body weight and food intake in rats submitted to caloric restrictions. PLoS One, 9(6):e100073, 2014.

[26] Terry Kenakin. Principles: receptor theory in pharmacology. Trends in pharmacological sciences, 25(4):186-192, Apr 2004.

[27] R. B. Lynn, G. Y. Cao, R. V. Considine, T. M. Hyde, and J. F. Caro. Autoradiographic localization of leptin binding in the choroid plexus of ob/ob and $\mathrm{db} / \mathrm{db}$ mice. Biochemical and Biophysical Research Communications, 219(3):884-889, Feb 1996. 
[28] R. L. Martin, E. Perez, Y. J. He, R. Dawson, and W. J. Millard. Leptin resistance is associated with hypothalamic leptin receptor mRNA and protein downregulation. Metabolism, 49(11):1479-1484, Nov 2000.

[29] G. J. Morton, D. E. Cummings, D. G. Baskin, G. S. Barsh, and M. W. Schwartz. Central nervous system control of food intake and body weight. Nature, 443(7109):289-295, Sep 2006.

[30] Gregory J Morton, Thomas H Meek, and Michael W Schwartz. Neurobiology of food intake in health and disease. Nature Reviews Neuroscience, 15(6):367-378, Jun 2014.

[31] Martin G Myers, Michael A Cowley, and Heike Münzberg. Mechanisms of leptin action and leptin resistance. Annual Review of Physiology, 70:537-556, 2008.

[32] Martin G Myers, Rudolph L Leibel, Randy J Seeley, and Michael W Schwartz. Obesity and leptin resistance: distinguishing cause from effect. Trends in Endocrinology \& Metabolism, 21(11):643-651, Nov 2010.

[33] J. Naukkarinen, A. Rissanen, J. Kaprio, and K. H. Pietiläinen. Causes and consequences of obesity: the contribution of recent twin studies. International Journal of Obesity, 36(8):10171024, Aug 2012.

[34] K. M. Nelson, R. L. Weinsier, C. L. Long, and Y. Schutz. Prediction of resting energy expenditure from fat-free mass and fat mass. The American journal of clinical nutrition, 56(5):848-856, Nov 1992.

[35] Rekha Pal and Abhiram Sahu. Leptin signaling in the hypothalamus during chronic central leptin infusion. Endocrinology, 144(9):3789-3798, Sep 2003.

[36] R. R. Ruffolo. Review important concepts of receptor theory. Journal of Autonomic Pharmacology, 2(4):277-295, Dec 1982.

[37] A. Sahu. Resistance to the satiety action of leptin following chronic central leptin infusion is associated with the development of leptin resistance in neuropeptide Y neurones. Journal of neuroendocrinology, 14(10):796-804, Oct 2002.

[38] P. J. Scarpace, M. Matheny, N. Tümer, K. Y. Cheng, and Y. Zhang. Leptin resistance exacerbates diet-induced obesity and is associated with diminished maximal leptin signalling capacity in rats. Diabetologia, 48(6):1075-1083, Jun 2005.

[39] M. W. Schwartz, E. Peskind, M. Raskind, E. J. Boyko, and D. Porte. Cerebrospinal fluid leptin levels: relationship to plasma levels and to adiposity in humans. Nature medicine, 2(5):589-593, May 1996.

[40] M. W. Schwartz, S. C. Woods, D. Porte, R. J. Seeley, and D. G. Baskin. Central nervous system control of food intake. Nature, 404(6778):661-671, Apr 2000.

[41] R. P. Stephenson. A modification of receptor theory. British journal of pharmacology and chemotherapy, 11(4):379-393, Dec 1956.

[42] Joshua Tam, Dai Fukumura, and Rakesh K Jain. A mathematical model of murine metabolic regulation by leptin: energy balance and defense of a stable body weight. Cell Metabolism, 9(1):52-63, Jan 2009.

[43] L. A. Tartaglia. The leptin receptor. The Journal of Biological Chemistry, 272(10):6093-6096, Mar 1997.

[44] B. Topp, K. Promislow, G. deVries, R. M. Miura, and D. T. Finegood. A model of beta-cell mass, insulin, and glucose kinetics: pathways to diabetes. Journal of Theoretical Biology, 206(4):605-619, Oct 2000.

[45] P. S. Widdowson, R. Upton, R. Buckingham, J. Arch, and G. Williams. Inhibition of food response to intracerebroventricular injection of leptin is attenuated in rats with diet-induced obesity. Diabetes, 46(11):1782-1785, Nov 1997.

[46] J. Wilsey and P. J. Scarpace. Caloric restriction reverses the deficits in leptin receptor protein and leptin signaling capacity associated with diet-induced obesity: role of leptin in the regulation of hypothalamic long-form leptin receptor expression. Journal of endocrinology, 181(2):297-306, May 2004. 
[47] A. M. Wren, L. J. Seal, M. A. Cohen, A. E. Brynes, G. S. Frost, K. G. Murphy, W. S. Dhillo, M. A. Ghatei, and S. R. Bloom. Ghrelin enhances appetite and increases food intake in humans. The Journal of Clinical Endocrinology \& Metabolism, 86(12):5992, Dec 2001.

[48] J. Zeng, B. W. Patterson, S. Klein, D. R. Martin, S. Dagogo-Jack, W. M. Kohrt, S. B. Miller, and M. Landt. Whole body leptin kinetics and renal metabolism in vivo. American Journal of Physiology - Endocrinology and Metabolism, 273(6 Pt 1):E1102-E1106, Dec 1997.

[49] Y. Zhang, M. Olbort, K. Schwarzer, B. Nuesslein-Hildesheim, M. Nicolson, E. Murphy, T. J. Kowalski, I. Schmidt, and R. L. Leibel. The leptin receptor mediates apparent autocrine regulation of leptin gene expression. Biochemical and Biophysical Research Communications, 240(2):492-495, Nov 1997.

[50] Yi Zhang and Philip J Scarpace. The role of leptin in leptin resistance and obesity. Physiology \&3 Behavior, 88(3):249-256, Jun 2006.

\section{A Analysis of a Simplified System}

\section{A.1 Model Formulation}

Let consider System (10), with all parameters being positive. Let's assume:

(H1) Variations of fat-free mass are negligible compared to variations of fat mass, so $d F F M / d F M=$ 0 . It follows that

$$
\Omega:=\gamma_{\Omega}(1+\alpha \exp (\kappa F M))=0, \quad F F M=F F M_{0},
$$

and the first equation of System (10) becomes

$$
\frac{d F M(t)}{d t}=\frac{\gamma_{E} F I(t)-\eta\left(\rho_{F M} F M(t)+\rho_{F F M} F F M_{0}+\xi\right)}{\rho_{F M}} .
$$

(H2) Following variations of the fat mass $F M$, leptin is instantaneously produced, proportionally to $F M$, so that

$$
L(t)=k_{L} F M(t), \quad \text { with } k_{L}=\frac{\gamma_{L}}{\delta_{L}} .
$$

Then, System (10) writes

$$
\left\{\begin{aligned}
\frac{d F M(t)}{d t} & =\tilde{\gamma}_{E} F I(t)-\eta F M(t)-\nu \\
\frac{d R(t)}{d t} & =\gamma_{R}\left(1+\tilde{\lambda}_{R, 1} F M(t)\right)-\delta_{R}\left(1+\tilde{\lambda}_{R, 2} F M^{2}(t)\right) R(t), \\
\frac{d F I(t)}{d t} & =\frac{\gamma_{F I}}{1+\phi_{R(t)}\left(k_{L} F M(t)\right)}-\delta_{F I} F I(t),
\end{aligned}\right.
$$

with

$$
\nu:=\frac{\eta\left(\rho_{F F M} F F M_{0}+\xi\right)}{\rho_{F M}}, \tilde{\gamma}_{E}:=\frac{\gamma_{E}}{\rho_{F M}}, \tilde{\lambda}_{R, 1}:=\lambda_{R, 1} k_{L}, \tilde{\lambda}_{R, 2}:=\lambda_{R, 2} k_{L}^{2} .
$$

For the sake of simplicity we will omit the tilde on parameter notations in the following.

We make the following additional assumptions:

(H3) Food intake is quasi constant (quasi steady state assumption) with $d F I(t) / d t=0$ and

$$
F I(t)=\frac{k_{F I}}{1+\phi_{R(t)}\left(k_{L} F M(t)\right)}, \quad k_{F I}:=\frac{\gamma_{F I}}{\delta_{F I}}>0 .
$$

(H4) The function $\phi_{R}(\cdot)$ is constant, with $\phi_{R}(L)=\phi R$.

Hence, System (20) becomes

$$
\left\{\begin{aligned}
\frac{d F M(t)}{d t} & =\gamma_{E} \frac{k_{F I}}{1+\phi R(t)}-\eta F M(t)-\nu \\
\frac{d R(t)}{d t} & =\gamma_{R}\left(1+\lambda_{R, 1} F M(t)\right)-\delta_{R}\left(1+\lambda_{R, 2} F M^{2}(t)\right) R(t) .
\end{aligned}\right.
$$


Assuming $\lambda_{R, 1} \gg \lambda_{R, 2}$, then a rescaling of System (21) gives (see A.3 for details)

$$
\left\{\begin{aligned}
\frac{d F M(t)}{d t} & =\frac{\gamma_{F M}}{1+\phi R(t)}-\eta F M(t)-\nu \\
\frac{d R(t)}{d t} & =\rho F M(t)-\delta_{R}\left(1+\lambda_{R} F M^{2}(t)\right) R(t),
\end{aligned}\right.
$$

where notations have been slightly modified, for the sake of simplicity. If $\lambda_{R 1}=0$, then the system (21) can be directly analyzed.

\section{A.2 Model Analysis}

Let search for steady states $(m, r)$ of System (22). They are constant solutions, satisfying

$$
\frac{\gamma_{F M}}{1+\phi r}=\eta m+\nu \quad \text { and } \quad r=\frac{\rho m}{\delta_{R}\left(1+\lambda_{R} m^{2}\right)} .
$$

Using the notation,

$$
\sigma:=\frac{\rho}{\delta_{R}}
$$

it follows, from (23),

$$
\eta m+\nu=\gamma_{F M} \frac{1+\lambda_{R} m^{2}}{1+\phi \sigma m+\lambda_{R} m^{2}} .
$$

Let define the functions $f_{1}$ and $f_{2}$ as follows:

$$
f_{1}(x):=\frac{\eta}{\gamma_{F M}} x+\frac{\nu}{\gamma_{F M}},
$$

and

$$
f_{2}(x):=\frac{1+\lambda_{R} x^{2}}{1+\mu x+\lambda_{R} x^{2}}, \quad \mu:=\phi \sigma .
$$

Let's focus on the function $f_{2}$. It equals 1 when $x=0$ and $x=+\infty$. Moreover,

$$
f_{2}^{\prime}(x)=\mu \frac{\lambda_{R} x^{2}-1}{\left(1+\mu x+\lambda_{R} x^{2}\right)^{2}}
$$

so $f_{2}$ is decreasing for $x<x_{1}$ and increasing for $x>x_{1}$, where $x_{1}:=\sqrt{1 / \lambda_{R}}$, with

$$
f_{2}\left(x_{1}\right)=\frac{2 \sqrt{\lambda_{R}}}{2 \sqrt{\lambda_{R}}+\mu} .
$$

In addition,

$$
f_{2}^{\prime \prime}(x)=2 \mu \frac{\mu+3 \lambda_{R} x-\lambda_{R}^{2} x^{3}}{\left(1+\mu x+\lambda_{R} x^{2}\right)^{3}}
$$

so there exists a unique $x_{2}>0$ such that $f_{2}^{\prime \prime}\left(x_{2}\right)=0$, with $0<x_{1}<x_{2}$ (one can easily check that $\left.x_{2}>\sqrt{3 / \lambda_{R}}\right)$. Hence, the function $f_{2}$ is positive, decreasing on the interval $\left[0, x_{1}\right]$, increasing on the interval $\left[x_{1},+\infty\right)$, convex on the interval $\left[0, x_{2}\right]$ and concave for $x>x_{2}$. We then reach the following conclusion (see Figure 10):

Proposition 1. Depending on the value of $\gamma_{F M}$, the problem $f_{1}(x)=f_{2}(x)$ may have 0 , 1 , or 3 solutions, which correspond to steady states $(m, r)$ of System (22):

Case 1 If $\gamma_{F M}<\nu$, then System (22) has no steady state;

Case 2 If $\gamma_{F M} \geq \nu$, and $\nu / \gamma_{F M} \approx 1$, then System (22) has only one steady state, with $(m, r) \approx$ $(0,0)$

Case 3 If $\gamma_{F M} \geq \nu$, and $\gamma_{F M}$ is large, then System (22) has only one steady state, with $(m, r) \approx$ $(+\infty, 0)$; 


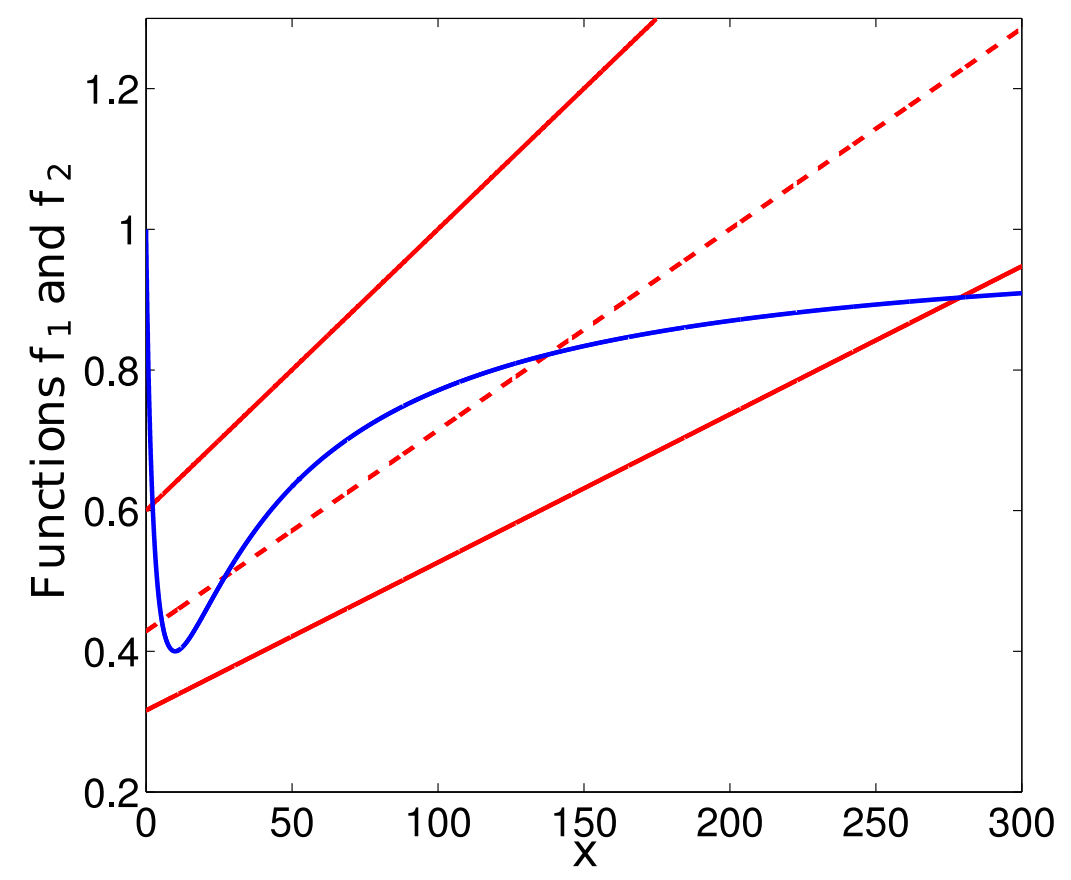

Figure 10: Graphs of the functions $f_{1}$ and $f_{2}$ defined in (24) and (25) for three different values of $\gamma_{F M}$, all satisfying $\gamma_{F M}>\nu$. The graph of function $f_{2}$ is displayed in blue, whereas functions $f_{1}$ are displayed in red. The top red line corresponds to $\gamma_{F M}=50$ and $\nu / \gamma_{F M}=0.7$, there is only one intersection between the two curves in the vicinity of $x=0$. The same occurs for the bottom red curve, corresponding to $\gamma_{F M}=95$, and the intersection occurs for $x$ large. The middle red dashed curve corresponds to $\nu / \gamma_{F M}=0.7$, and is associated with three intersections (see Proposition 1).

Case 4 If $\gamma_{F M} \geq \nu$, with $\gamma_{F M} \in\left(\gamma_{\min }, \gamma_{\max }\right)$ (values $\gamma_{\min }$ and $\gamma_{\max }$ are to be determined and depend on other parameter values), then System (22) has 3 steady states, denoted by $\left(m_{l}, r_{l}\right)$, $\left(m_{m}, r_{m}\right)$, and $\left(m_{h}, r_{h}\right)$ respectively, such that $m_{l}<m_{m}<m_{h}, f_{1}^{\prime}\left(m_{l}\right)>f_{2}^{\prime}\left(m_{l}\right), f_{1}^{\prime}\left(m_{m}\right)<$ $f_{2}^{\prime}\left(m_{m}\right)$, and $f_{1}^{\prime}\left(m_{h}\right)>f_{2}^{\prime}\left(m_{h}\right)$.

Proof. Here are some hints for each case. Case 1: the function $f_{1}$ is an increasing function satisfying $f_{1}(0)>1 \geq f_{2}(x)$ for all $x \geq 0$. Case 2 : The function $f_{1}$ becomes larger than 1 for $x$ close to 0 so the problem $f_{1}(x)=f_{2}(x)$ has only one solution ( $f_{1}$ is increasing, $f_{2}$ is decreasing) in the vicinity of $x=0$. Case 3 : The function $f_{1}$ is flatter, yet goes towards infinity, and then crosses the bounded function $f_{2}$ for large values of $x$. Case 4: This case is illustrated on Figure 10.

Let's now focus on the linear stability of the steady states of System (22). Let denote by $(m, r)$ a steady state of $(22)$. Linearization of $(22)$ around $(m, r)$ leads to

$$
\left\{\begin{aligned}
\frac{d F M(t)}{d t} & =-a(r) R(t)-\eta F M(t), \\
\frac{d R(t)}{d t} & =b(m, r) F M(t)-c(m) R(t),
\end{aligned}\right.
$$

where

$$
\begin{array}{rlr}
a(r) & =\frac{\gamma_{F M} \phi}{(1+\phi r)^{2}} \quad>0, \\
b(m, r) & =\rho-2 \lambda_{R} \delta_{R} m r, \\
c(m) & =\delta_{R}\left(1+\lambda_{R} m^{2}\right) \quad>0 .
\end{array}
$$


Hence, $(m, r)$ is locally asymptotically stable if and only if

$$
-\eta-c(m)<0
$$

and

$$
\eta c(m)+a(r) b(m, r)>0 .
$$

The first condition is straightforwardly satisfied due to the positivity of $c(m)$. The second condition writes

$$
\eta \delta_{R}\left(1+\lambda_{R} m^{2}\right)+\frac{\gamma_{F M} \phi}{(1+\phi r)^{2}}\left(\rho-2 \lambda_{R} \delta_{R} m r\right)>0 .
$$

Using (23), Inequality (26) is equivalent to

$$
H(m)>0
$$

where

$$
H(m):=\eta\left(1+\mu m+\lambda_{R} m^{2}\right)^{2}-\gamma_{F M} \mu\left(\lambda_{R} m^{2}-1\right) .
$$

It follows that $H(m)>0$ if and only if

$$
f_{2}^{\prime}(m)<\frac{\eta}{\gamma_{F M}}=f_{1}^{\prime}(m)
$$

We can then conclude to the stability of System (22) in the next Proposition.

Proposition 2. When System (22) has only one steady state, then it is locally asymptotically stable. When System $(22)$ has 3 steady states, then the steady states $\left(m_{l}, r_{l}\right)$ and $\left(m_{h}, r_{h}\right)$, associated with the lower and higher values of $m$, respectively (see Proposition 1), are locally asymptotically stable whereas the intermediate steady state $\left(m_{m}, r_{m}\right)$ is unstable: System (22) is bistable.

\section{A.3 Rescaling of System (21)}

Consider System (21), and set

$$
m(t):=\frac{\lambda_{R, 1} F M(t)+1}{\sqrt{\lambda_{R, 1}^{2}+\lambda_{R, 2}}}, \quad r(t):=R(t) .
$$

Then $m$ and $r$ satisfy

$$
\left\{\begin{aligned}
m^{\prime}(t) & =\frac{\gamma_{m}}{1+\phi r(t)}-\eta m(t)-\nu_{m} \\
r^{\prime}(t) & =\gamma_{r} m(t)-\delta_{r}\left(1-2 \frac{\lambda_{R, 2}}{\sqrt{\lambda_{R, 1}^{2}+\lambda_{R, 2}}} m(t)+\lambda_{R, 2} m^{2}(t)\right) r(t)
\end{aligned}\right.
$$

where

$$
\begin{aligned}
\gamma_{m}:=\gamma_{E} k_{F I} \frac{\lambda_{R, 1}}{\sqrt{\lambda_{R, 1}^{2}+\lambda_{R, 2}}}, & \nu_{m}:=\frac{\lambda_{R, 1} \nu-\eta}{\sqrt{\lambda_{R, 1}^{2}+\lambda_{R, 2}}}, \\
\gamma_{r}:=\gamma_{R} \sqrt{\lambda_{R, 1}^{2}+\lambda_{R, 2}}, & \delta_{r}:=\delta_{R} \frac{\lambda_{R, 1}^{2}+\lambda_{R, 2}}{\lambda_{R, 1}^{2}} .
\end{aligned}
$$

Since we assumed $\lambda_{R, 1} \gg \lambda_{R, 2}$, then

$$
\frac{\lambda_{R, 2}}{\sqrt{\lambda_{R, 1}^{2}+\lambda_{R, 2}}} \approx 0
$$

and

$$
\begin{array}{ll}
\gamma_{m} \approx \gamma_{E} k_{F I}=\gamma_{F M}, & \nu_{m} \approx \nu-\frac{\eta}{\lambda_{R, 1}}, \\
\gamma_{r} \approx \gamma_{R} \lambda_{R, 1}, & \delta_{r} \approx \delta_{R} .
\end{array}
$$

One obtains System (22). 